\title{
Molecular Line Emission Towards High-Mass Clumps: The MALT90 Catalogue
}

\author{
J. M. Rathborne ${ }^{1,12}$, J. S. Whitaker ${ }^{2}$, J. M. Jackson ${ }^{3}$, J. B. Foster ${ }^{4}$, Y. Contreras ${ }^{1,5}$, I. W. Stephens ${ }^{3}$, \\ A. E. Guzmán ${ }^{6}$, S. N. Longmore ${ }^{7}$, P. Sanhueza ${ }^{8}$, F. Schuller ${ }^{9}$, F. Wyrowski ${ }^{10}$ and J. S. Urquhart ${ }^{10,11}$ \\ ${ }^{1}$ CSIRO Astronomy and Space Science, PO Box 76, Epping, NSW 1710, Australia \\ ${ }^{2}$ Physics Department, Boston University, Boston, MA 02215, USA \\ ${ }^{3}$ Institute for Astrophysical Research, Boston University, Boston, MA 02215, USA \\ ${ }^{4}$ Department of Astronomy, Yale University, P.O. Box 208101, New Haven, CT 06520-8101, USA \\ ${ }^{5}$ Leiden Observatory, Leiden University, PO Box 9513, NL-2300 RA, Leiden, the Netherlands \\ ${ }^{6}$ Departamento de Astronomía, Universidad de Chile, Camino el Observatorio 1515, Las Condes, Santiago, Chile \\ ${ }^{7}$ Astrophysics Research Institute, Liverpool John Moores University, 146 Brownlow Hill, Liverpool L3 5RF, UK \\ ${ }^{8}$ National Astronomical Observatory of Japan, 2-21-1- Osawa, Mitaka, Tokyo 181-8588, Japan \\ ${ }^{9}$ European Southern Observatory, Alonso de Cordova 3107, Vitacura, Santiago, Chile \\ ${ }^{10}$ Max Planck Institute for Radioastronomy, Auf dem Hugel 69, 53121, Bonn, Germany \\ ${ }^{11}$ Centre for Astrophysics and Planetary Science, University of Kent, Canterbury, CT2 7NH, UK \\ ${ }^{12}$ Email: Jill.Rathborne@ csiro.au
}

(Received March 19, 2016; AcCePted April 29, 2016)

\begin{abstract}
The Millimetre Astronomy Legacy Team $90 \mathrm{GHz}$ survey aims to characterise the physical and chemical evolution of high-mass clumps. Recently completed, it mapped $90 \mathrm{GHz}$ line emission towards 3246 high-mass clumps identified from the ATLASGAL $870 \mu \mathrm{m}$ Galactic plane survey. By utilising the broad frequency coverage of the Mopra telescope's spectrometer, maps in 16 different emission lines were simultaneously obtained. Here, we describe the first catalogue of the detected line emission, generated by Gaussian profile fitting to spectra extracted towards each clumps' $870 \mu \mathrm{m}$ dust continuum peak. Synthetic spectra show that the catalogue has a completeness of $>95 \%$, a probability of a false-positive detection of $<0.3 \%$, and a relative uncertainty in the measured quantities of $<20 \%$ over the range of detection criteria. The detection rates are highest for the (1-0) transitions of $\mathrm{HCO}^{+}, \mathrm{HNC}, \mathrm{N}_{2} \mathrm{H}^{+}$, and $\mathrm{HCN}(\sim 77-89 \%)$. Almost all clumps $(\sim 95 \%)$ are detected in at least one of the molecular transitions, just over half of the clumps $(\sim 53 \%)$ are detected in four or more of the transitions, while only one clump is detected in 13 transitions. We find several striking trends in the ensemble of properties for the different molecular transitions when plotted as a function of the clumps' evolutionary state as estimated from Spitzer mid-IR images, including (1) HNC is relatively brighter in colder, less evolved clumps than those that show active star formation, (2) $\mathrm{N}_{2} \mathrm{H}^{+}$is relatively brighter in the earlier stages, (3) that the observed optical depth decreases as the clumps evolve, and (4) the optically thickest $\mathrm{HCO}^{+}$emission shows a 'blue-red asymmetry' indicating overall collapse that monotonically decreases as the clumps evolve. This catalogue represents the largest compiled database of line emission towards high-mass clumps and is a valuable data set for detailed studies of these objects.
\end{abstract}

Keywords: ISM: clouds - ISM: molecules - stars: formation - catalogs

\section{INTRODUCTION}

High-mass stars $\left(>8 \mathrm{M}_{\odot}\right)$ typically form in clusters from dense molecular clumps with sizes of $\sim 1$ pc and masses of $\gtrsim 200 \mathrm{M}_{\odot}$. Recent infrared and millimetre/submillimetre Galactic plane surveys have identified a large number of dense molecular clumps in all stages of evolution (e.g., Benjamin et al. 2003; Churchwell et al. 2009; Carey et al. 2009;

*Data for this paper is deposited here: http://dx.doi.org/10.4225/50/ 57635C7B4F722.
Molinari et al. 2010; Schuller et al. 2009; Aguirre et al. 2011). Complemented by radio continuum surveys (e.g., Hoare et al. 2012; Purcell et al. 2013), surveys targeting ammonia and water/methanol masers (e.g., Green et al. 2009; Caswell et al. 2010; Walsh et al. 2011; Purcell et al. 2012), and those pinpointing massive young stellar objects (MYSO) and $\mathrm{H}$ II regions (e.g., Hoare et al. 2005; Urquhart et al. 2008; Lumsden et al. 2013), these large datasets have enabled significant progress towards improving our understanding of star formation within the Galaxy. Indeed, the combination of these 
datasets have allowed detailed studies, in particular, towards the latter phases in evolution where high-mass protostars and H II regions have formed (e.g., Urquhart et al. 2013, 2014b; Tremblay et al. 2015; Csengeri et al. 2016).

The Millimetre Astronomy Legacy Team $90 \mathrm{GHz}$ (MALT90) survey builds on previous studies and aims to characterise the global conditions within, and evolution of, the dense clumps from which high-mass stars form. MALT90, by tracing their dense gas, will determine the physical and chemical evolution within $\sim 3000$ high-mass clumps located throughout the Galaxy (Foster et al. 2011; Jackson et al. 2013; Foster et al. 2013). While detailed studies often focus on a particular stage in the evolution of high-mass clump, the MALT90 survey includes clumps in all stages of evolution, from the cold, starless clumps devoid of star formation to those with high-mass stars and embedded $\mathrm{H}_{\text {II }}$ regions. As such, MALT90 provides a legacy database for the study of high-mass star formation.

The survey can be utilised to characterise the ensemble properties and evolution of high-mass clumps and also to identify unusual or extreme clumps within the population. Indeed, several studies have already begun to utilise the survey for these purposes (e.g., Hoq et al. 2013; Liu, Wang, \& Xu 2013; Miettinen 2014; Rathborne et al. 2014a; He et al. 2015; Stephens et al. 2015; Yu \& Wang 2015; Contreras et al. 2016; Stephens et al., submitted). In addition, MALT90 also provides a definitive source list for detailed ALMA observations aimed at understanding the evolution of high-mass clumps. Indeed, ALMA cycle 0 observations towards a highmass MALT90 clump, G0.253+0.016 (also known as 'the Brick'), shows remarkable structure on small spatial scales (Rathborne et al. 2014b, 2015) and reveals details about mass assembly before star formation has begun.

Here, we report the properties of the molecular line emission detected towards the $870 \mu \mathrm{m}$ dust continuum peak of each clump observed as part of the MALT90 survey determined via Gaussian profile fitting to the spectra. Characterising their molecular line emission is the foundation for a number of scientific investigations, including the determination of kinematic distances and investigations of the physical, kinematic, and chemical states of high-mass clumps. Figures 1-8 provide an overview of the APEX Telescope Large Area Survey of the Galaxy (ATLASGAL)/Spitzer images and MALT90 spectra towards selected clumps contained within the catalogue. These figures are included to show examples of the Gaussian profile fitting procedures and reported catalogue values that are described in the following sections. We discuss these in more detail in Section 6.

\section{THE MALT90 SURVEY}

Using the 22-m Mopra telescope, MALT90 obtained 3 arcmin $\times 3$ arcmin maps towards 2014 locations along the Galactic plane. Jackson et al. (2013) provides the full details of the survey strategy, observing procedure, mapping param- eters, and data reduction. ${ }^{1}$ The map locations were selected to cover clumps identified from the ATLASGAL $870 \mu \mathrm{m}$ dust continuum emission survey (Schuller et al. 2009).

Schuller et al. (2009) estimate that, for cold dust with $T_{\text {dust }}=10 \mathrm{~K}$, the ATLASGAL $870 \mu \mathrm{m}$ peak flux sensitivity of $0.25 \mathrm{Jy}$ corresponds to a clump mass of $\sim 450 \mathrm{M}_{\odot}$ at a distance of $10 \mathrm{kpc}$. MALT90 used this flux sensitivity to select clumps in the Galactic Longitude range of -60 to $+20^{\circ}$ from the ATLASGAL catalogues of Contreras et al. (2013) and Urquhart et al. (2014a). In addition to this flux sensitivity limit, we further imposed criteria based on the clumps' level of IR emission (to ensure that clumps in all stages of evolution were included within the survey) and gave preference to those located in the Galaxy's fourth quadrant.

By utilising the broad frequency coverage of the Mopra telescope's spectrometer, maps in 16 different emission lines near $90 \mathrm{GHz}$ were obtained simultaneously. The transitions covered by the MALT90 survey (see Table 1 for details) were selected to be unambiguous probes of the dense clumps, located within larger, more diffuse giant molecular clouds (GMCs), that will give rise to stars. Because the $90 \mathrm{GHz}$ molecules require high volume densities for their excitation $\left(10^{5}-10^{6} \mathrm{~cm}^{-3}\right)$ and span a range in their excitation energies (5-50 K; Müller et al. 2001, 2005; Schöier et al. 2005), they are excellent tracers of distinct physical conditions. Moreover, they also probe distinct stages of chemical evolution. Since the chemistry evolves quite substantially from the cold gas phase to the hot gas phase around a newly formed highmass star, the presence or absence of emission from these molecules may also indicate distinct stages of chemical evolution.

Now complete, MALT90 is the largest database of molecular line emission towards high-mass clumps. Because the maps have good spatial (38 arcsec) and excellent spectral $\left(0.11 \mathrm{~km} \mathrm{~s}^{-1}\right)$ resolution, they reveal a wealth of information about the clump morphologies, chemistry, and kinematics. This catalogue is a preliminary investigation to characterise the molecular line emission towards this large sample of clumps.

\section{CHARACTERISING STAR FORMATION WITHIN EACH CLUMP}

To describe the IR signatures of the star-formation activity within each clump, Spitzer images (3-24 $\mu \mathrm{m}$ ) were used to classify each into five broad categories: 'quiescent' clumps (these are cold and dense and will be IR dark), 'protostellar' clumps (these contain an accreting protostar and will be associated with either extended $4.5 \mu \mathrm{m}$ emission, indicating shocked gas, or a compact $24 \mu \mathrm{m}$ point source, tracing the warm dust surrounding the embedded star), 'H II regions'

\footnotetext{
${ }^{1}$ All MALT90 raw data, processed cubes and moment maps are publicly available through the Australia Telescope Online Archive (ATOA; http://atoa.atnf.csiro.au/).
} 


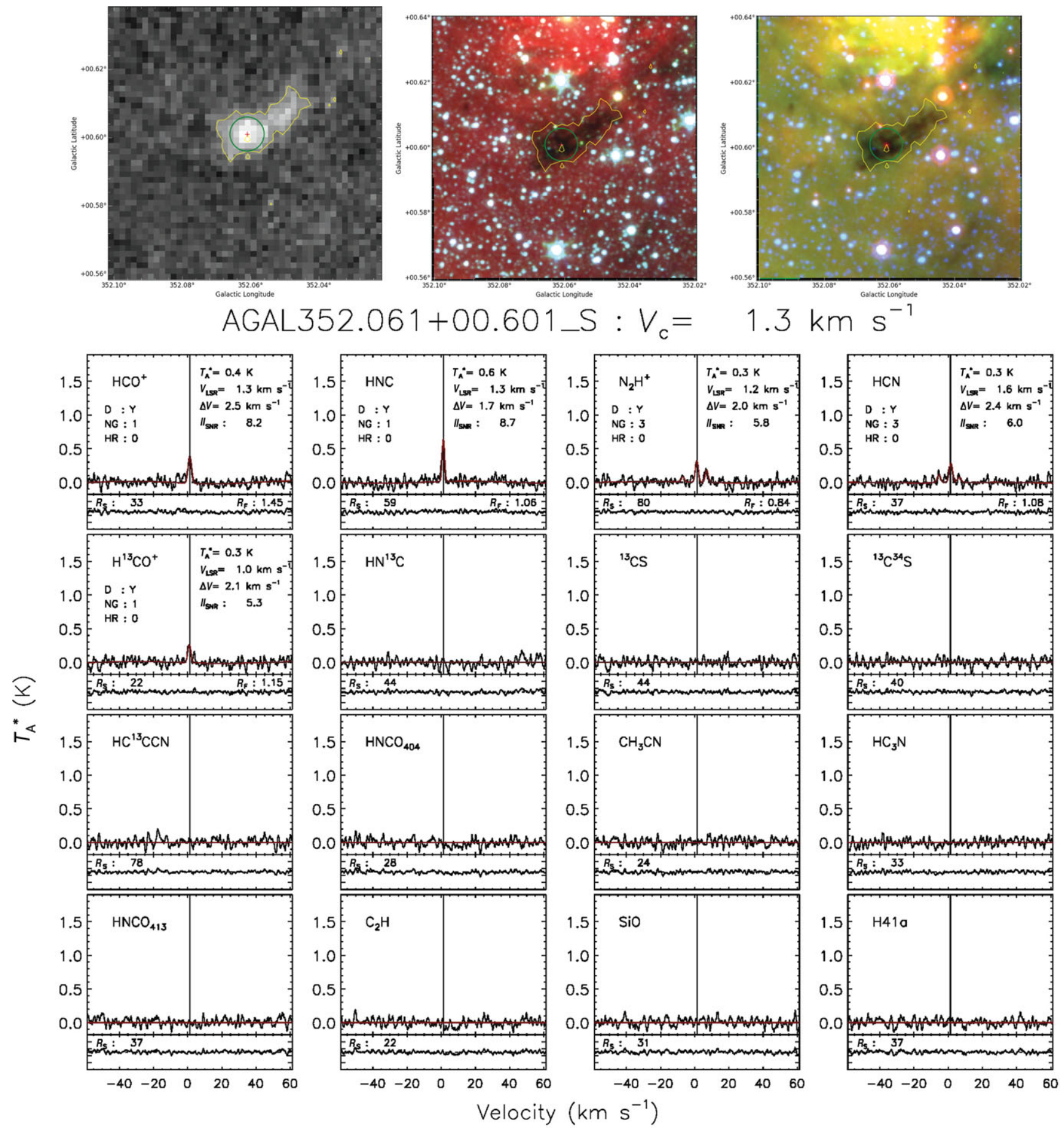

Figure 1. ATLASGAL/Spitzer images and MALT90 spectra towards AGAL352.061+00.601_S : an example of a 'quiescent' clump. Upper images (from left to right): ATLASGAL $870 \mu \mathrm{m}$ dust continuum emission in grey-scale, Spitzer 3-8 $\mu \mathrm{m}$ three colour image (3.6 $\mu \mathrm{m}$ in blue, $4.5 \mu \mathrm{m}$ in green, and $8.0 \mu \mathrm{m}$ in red), and Spitzer 3-24 $\mu \mathrm{m}$ three colour image (3.6 $\mu \mathrm{m}$ in blue, $8.0 \mu \mathrm{m}$ in green, and $24 \mu \mathrm{m}$ in red). In all images, yellow contours show the $870 \mu \mathrm{m}$ dust continuum emission. The red cross marks the peak of the ATLASGAL dust clump towards which the MALT90 spectra were extracted (any other ATLASGAL clumps located nearby are marked as blue crosses; they are listed as separate entries in the MALT90 catalogue). The green circle shows the angular resolution of the MALT90 data ( $\sim 38$ arcsec). Lower panels: MALT90 spectra extracted towards this ATLASGAL clump. The panels show the individual spectra (black) overlaid with the best-fitting profile (red; which includes any additional baseline component). If emission was detected, the derived parameters from the best-fitting Gaussian profile are labelled. The consensus velocity is labelled and shown in all panels as the solid vertical line. Under each panel, the residual spectrum is displayed with labels showing the residual in the complete spectrum $\left(R_{\mathrm{s}}\right)$ and the residual in the fit range $\left(R_{\mathrm{f}}\right)$. 

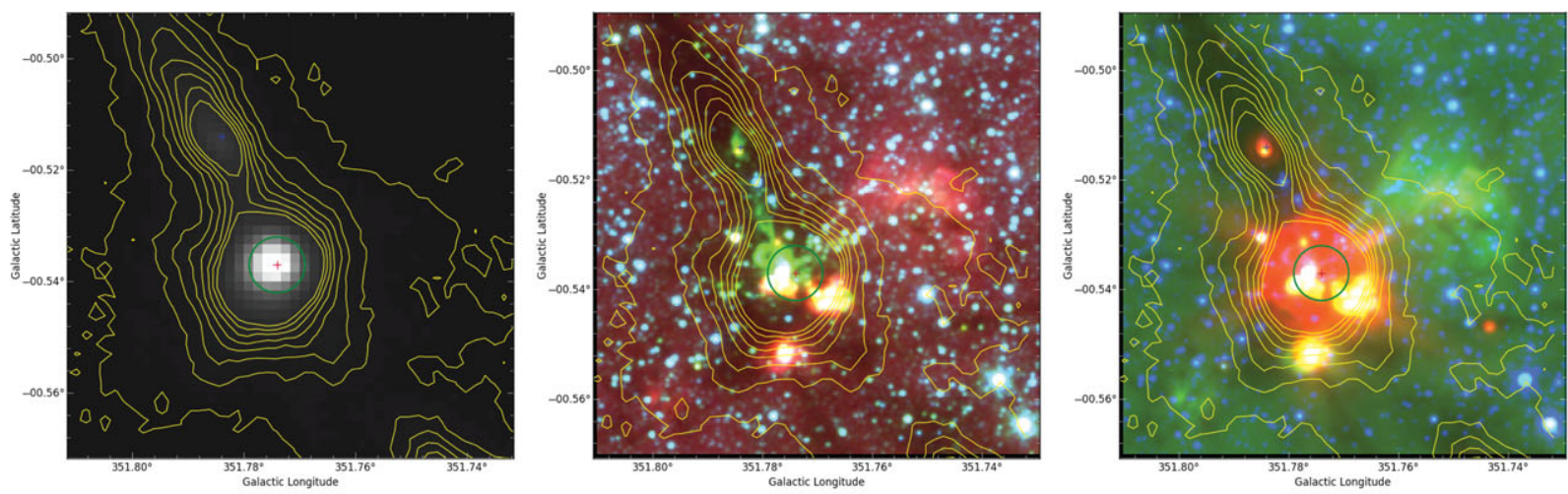

$$
\text { AGAL351.774-00.537_S : } V_{c}=-2.1 \mathrm{~km} \mathrm{~s}^{-1}
$$
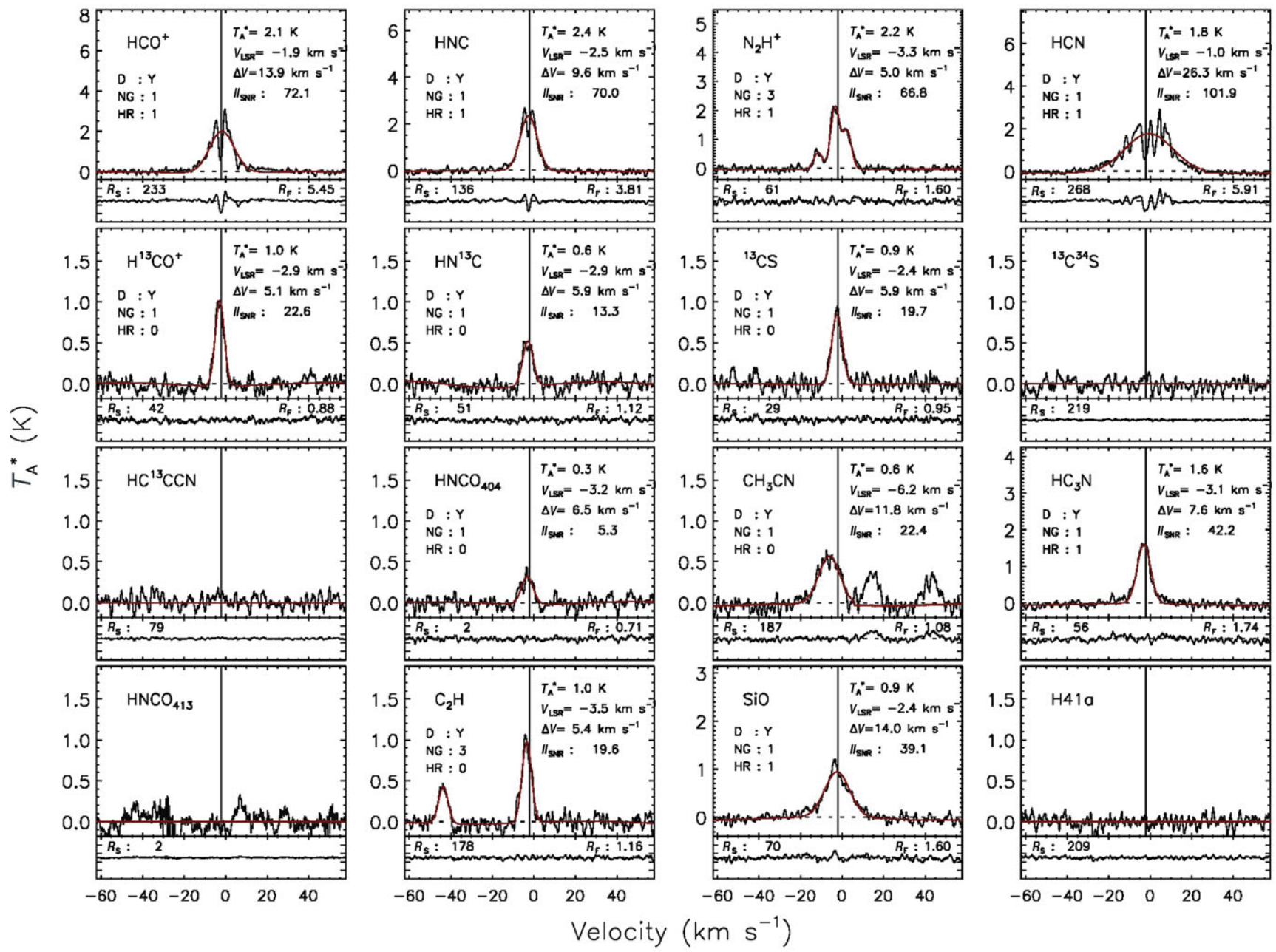

Figure 2. ATLASGAL/Spitzer images and MALT90 spectra towards AGAL351.774-00.537_S: an example of a 'protostellar' clump associated with bright and extended 'green' $(4.5 \mu \mathrm{m})$ emission, indicative of shocked gas. The images and spectra are the same as plotted and described in Figure 1.

(since the ionising radiation from an $\mathrm{H}_{\mathrm{II}}$ region will heat the gas and dust around the newly formed star, these will be IR bright and either compact or extended), and 'PDRs' (Photodominated regions; dominated by $8 \mu \mathrm{m}$ emission attributed to polycyclic aromatic hydrocarbons (PAHs), these occur at the molecular/ionised gas interface and are excited by the UV radiation from a nearby high-mass star).
We also use an additional category (referred to as 'uncertain') to separate out clumps for which their IR signatures are obscured by foreground emission or are ambiguous. Since the optically thin dust emission from ATLASGAL traces all of the material along the line of sight across the Galaxy but Spitzer is most sensitive to nearby emission, many of these 'uncertain' clumps may in fact lie at the far 

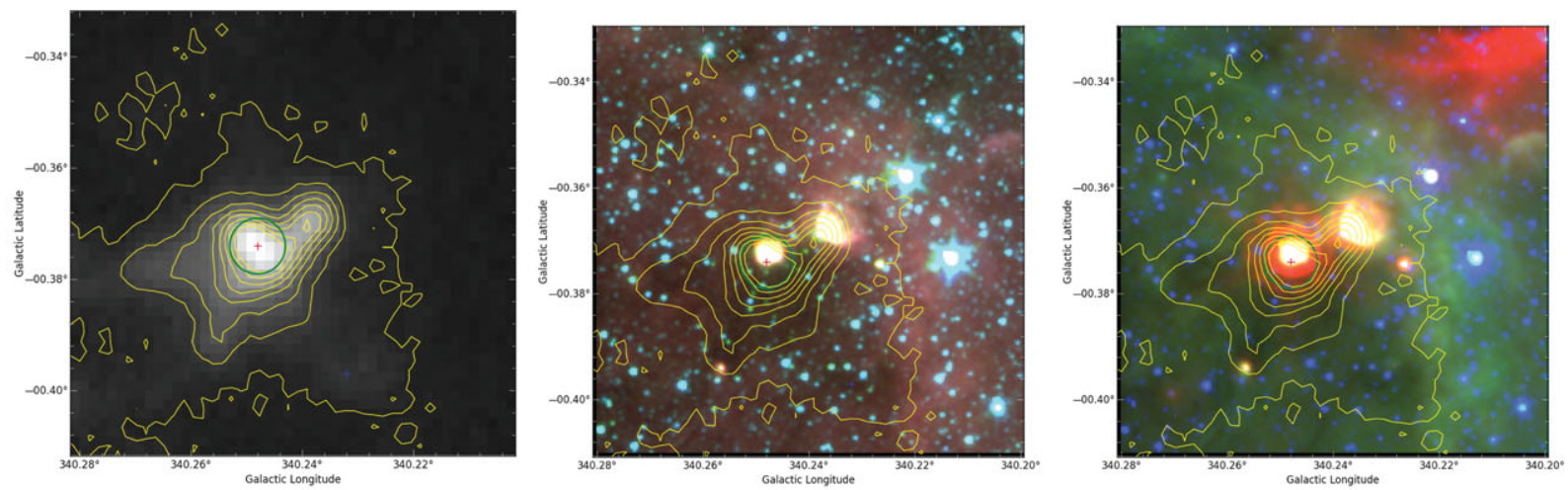

AGAL340.248-00.374_S : $V_{\mathrm{c}}=-50.4 \mathrm{~km} \mathrm{~s}^{-1}$
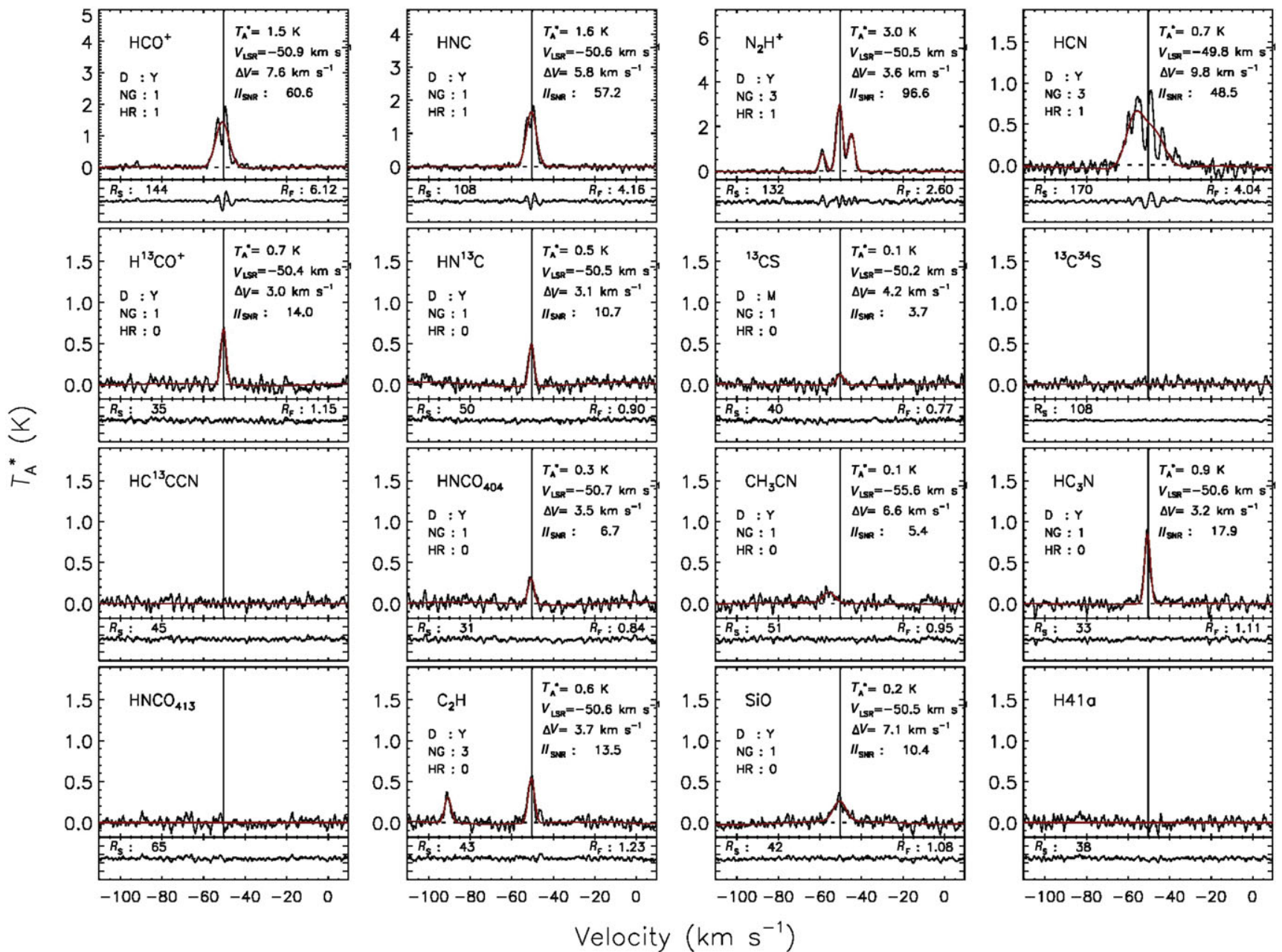

Figure 3. ATLASGAL/Spitzer images and MALT90 spectra towards AGAL340.248-00.374_S: an example of an 'HII' region. The images and spectra are the same as plotted and described in Figure 1.

side of the Galaxy. For simplicity and to ensure that these IR-based categories contain only clumps for which IR signatures (either dark or bright) are clearly associated, we exclude the 'uncertain' clumps from any analysis based on these IR categories.

Within the survey we classify 616 clumps as 'quiescent' (Q), 753 as 'protostellar' (A), 171 as compact $\mathrm{H}_{\text {II }}$ regions
(C), 688 as extended 'H II regions' (H), 345 as 'PDRs' (P), and 673 as 'uncertain' (U).

Recognising that this scheme separates clumps into distinct categories based on their IR emission alone, we we refer to these as 'IR-based' categories. Moreover, the assignment of clumps to these categories is somewhat arbitrary since the underlying evolution of the clumps is a continuum process 

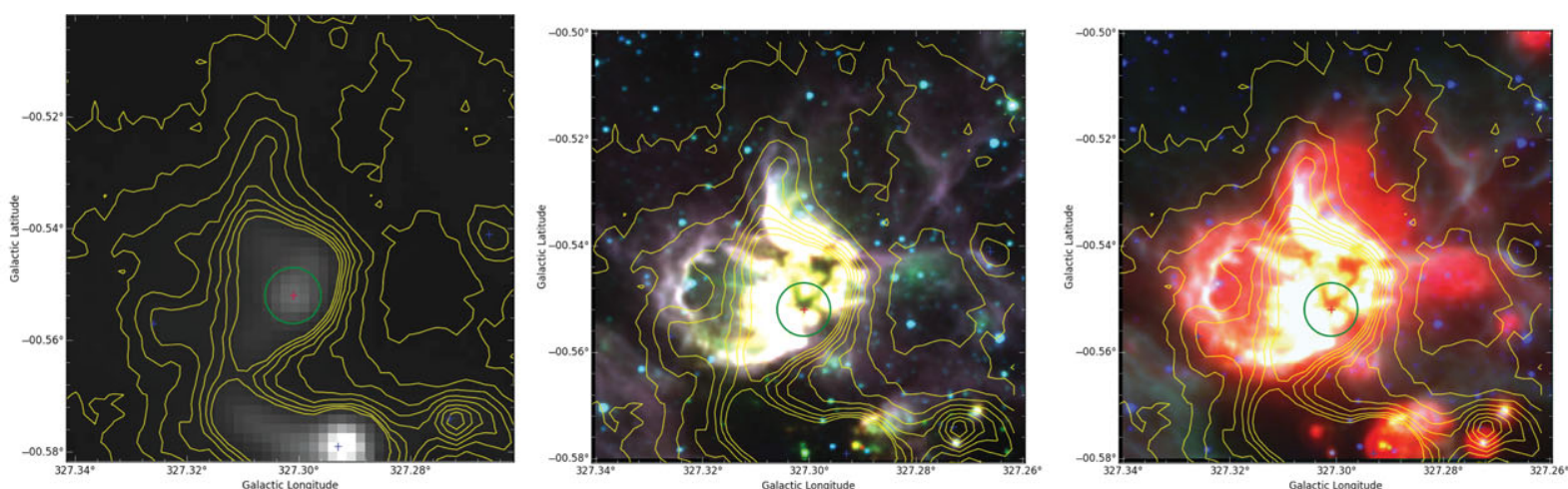

AGAL327.301-00.552_S : $V_{c}=-46.9 \mathrm{~km} \mathrm{~s}^{-1}$
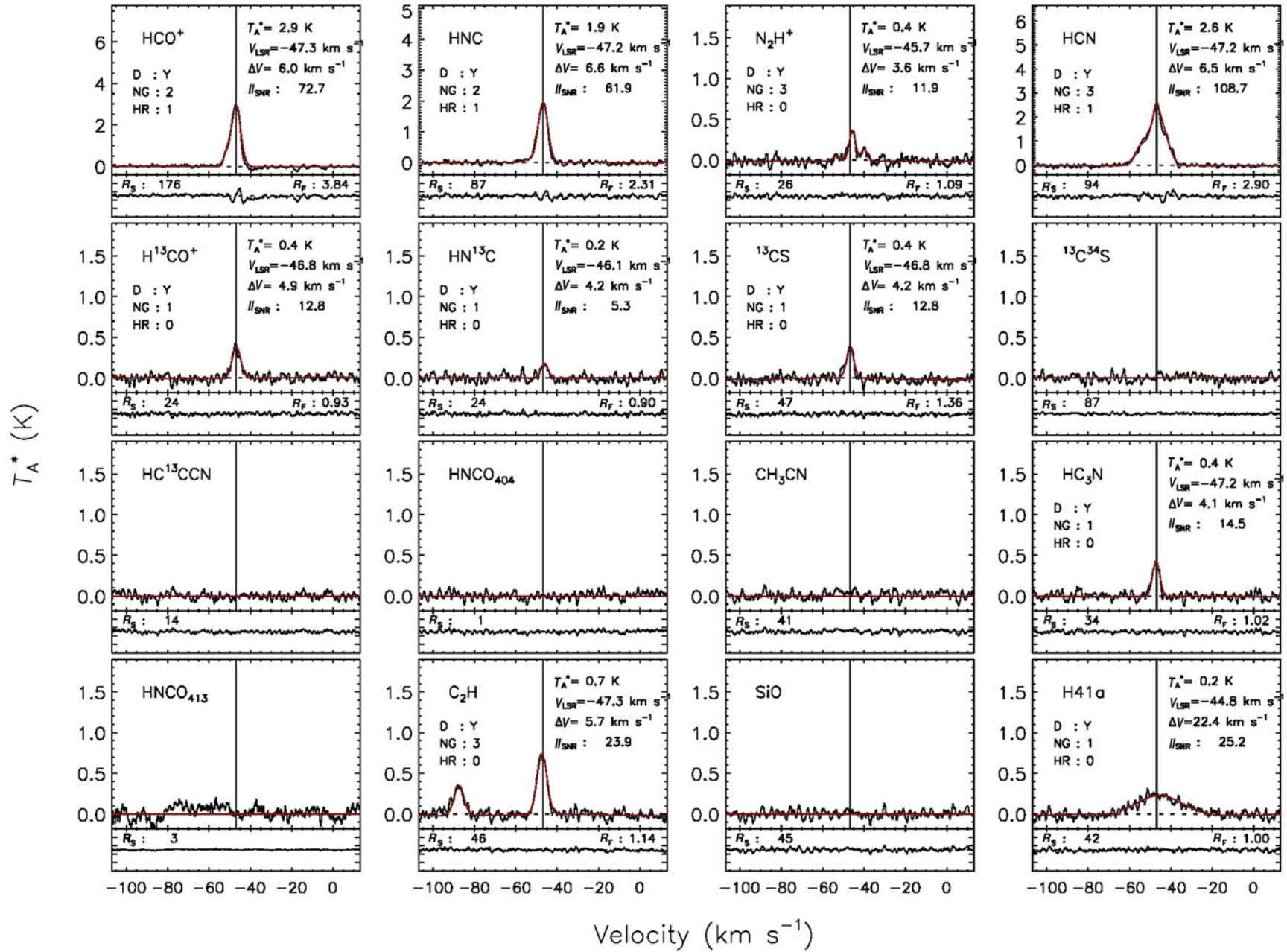

Velocity $\left(\mathrm{km} \mathrm{s}^{-1}\right)$

Figure 4. ATLASGAL/Spitzer images and MALT90 spectra towards AGAL327.301-00.552_S: an example of an 'HII' region, with a clear detection of $\mathrm{H} 41 \alpha$ emission. The images and spectra are the same as plotted and described in Figure 1.

and, as such, not easily defined into distinct phases. Nevertheless, we use this IR-based categorisation scheme to separate those clumps that are clearly associated with high-mass stars (i.e., those associated with $\mathrm{H}_{\text {II }}$ regions) from those that show evidence for early stages of embedded star formation (i.e., protostellar) or no evidence of embedded star formation (i.e., quiescent) and from those associated with PDRs.
In a similar manner to previous work, the identification and characterisation of any associated radio continuum and/or maser emission would complement and further refine these categories. This detailed analysis will be done in subsequent papers.

The reliability of this IR-based classification scheme to indicate clump evolution has been investigated by Guzmán 

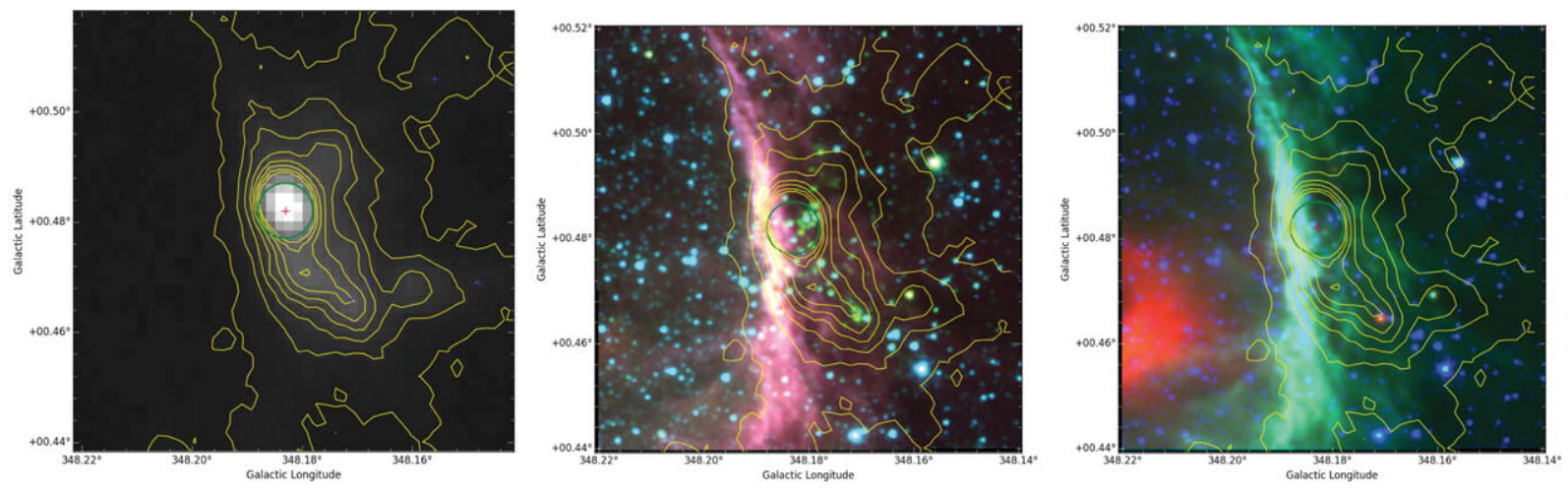

AGAL348.183+00.482_S: $V_{c}=-6.6 \mathrm{~km} \mathrm{~s}^{-1}$
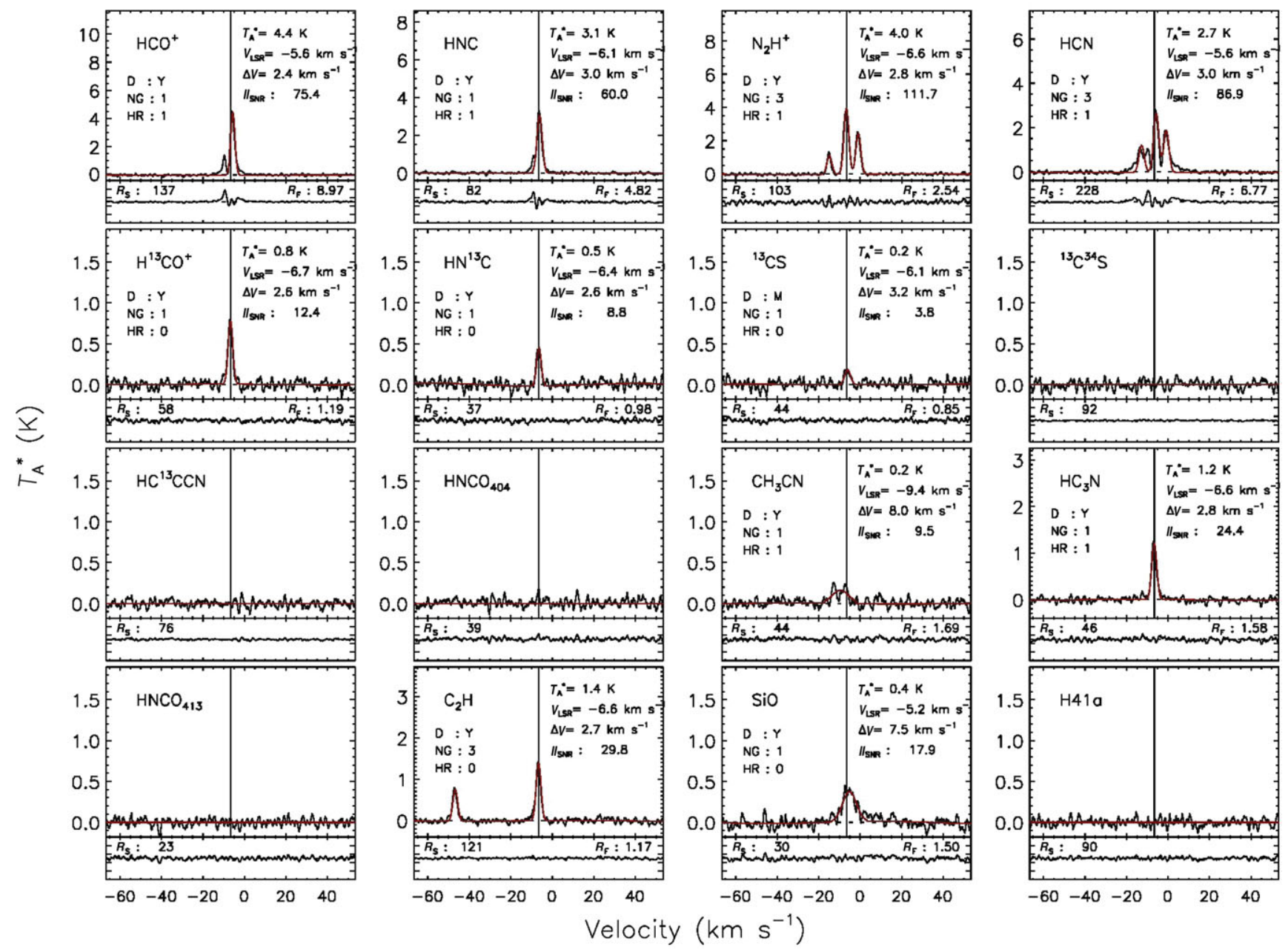

Figure 5. ATLASGAL/Spitzer images and MALT90 spectra towards AGAL348.183+00.482_S : an example of a 'PDR' clump. The images and spectra are the same as plotted and described in Figure 1.

et al. (2015). Using Herschel data, Guzmán et al. (2015) found that the average dust temperature for each category increased monotonically from the quiescent clumps, to the protostellar clumps, to those associated with $\mathrm{H}_{\text {II }}$ regions. Moreover, the column density varies significantly among the categories, increasing from quiescent to protostellar and then decreasing in subsequent stages. These trends show that the
IR-based categories do in fact reveal significant differences in the clump population and give us confidence that these categories reliably indicate clump evolution. Thus, despite the caveats, this scheme can be used to describe the evolutionary state of the molecular clumps.

Since these IR-based categories broadly represent the stages in evolution for a clump with increasing levels 

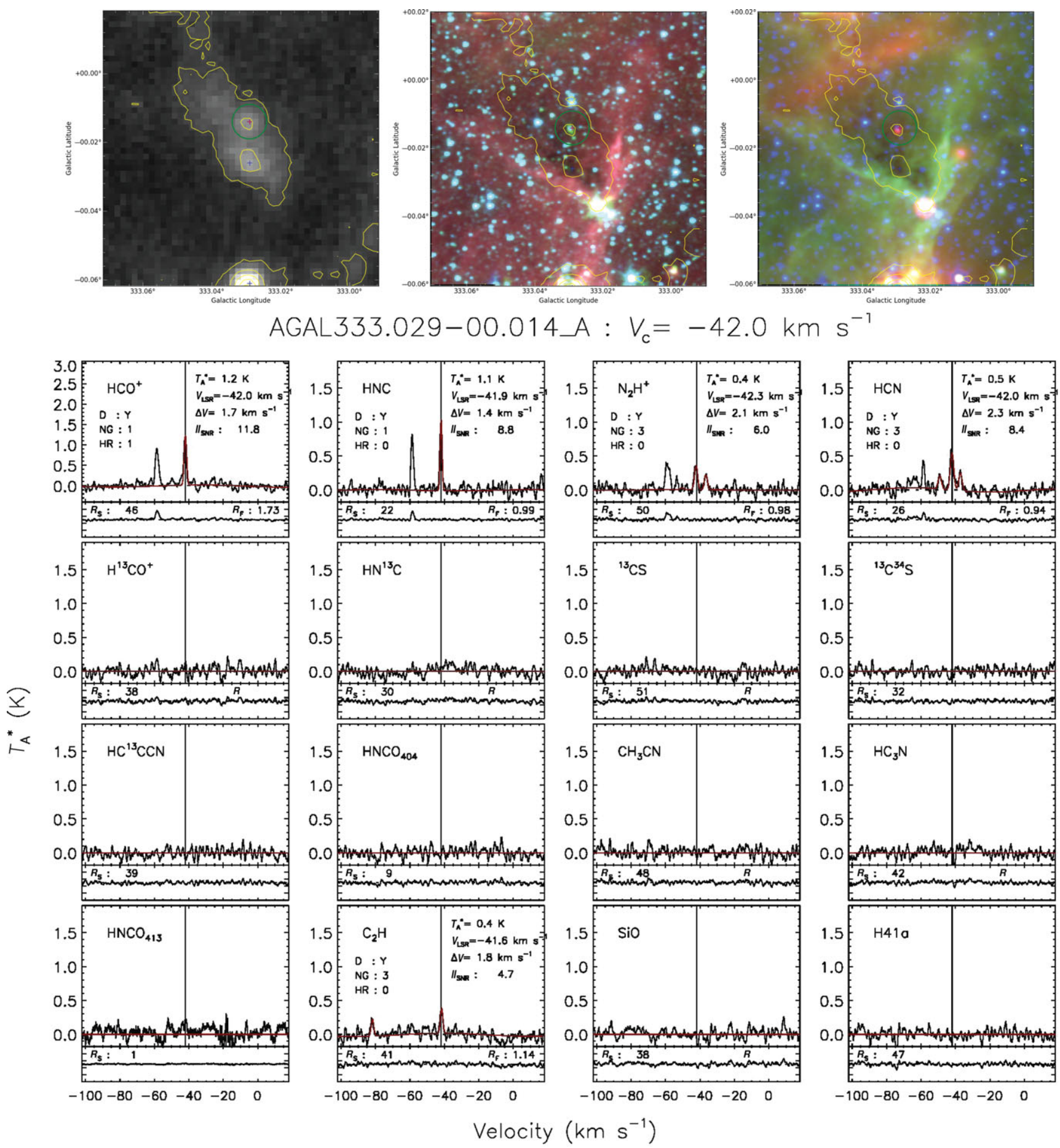

Figure 6. ATLASGAL/Spitzer images and MALT90 spectra towards AGAL333.029-00.014_A : an example of a 'Protostellar' clump with two velocity components detected along the line of sight (component A is shown here, component B is shown in Figure 7). The images and spectra are the same as plotted and described in Figure 1.

of star formation, we use them as a proxy for evolution: quiescent clumps represent an early phase, protostellar clumps show the initial signatures of active star formation, while clumps associated with $\mathrm{H}_{\text {II }}$ regions represent a later stage when young, high-mass stars have already formed.

\section{CHARACTERISING LINE EMISSION WITHIN EACH CLUMP}

Mapping each of the ATLASGAL clumps was required in order to address many of the science goals of the MALT90 survey. These maps reveal that the spatial distribution of 

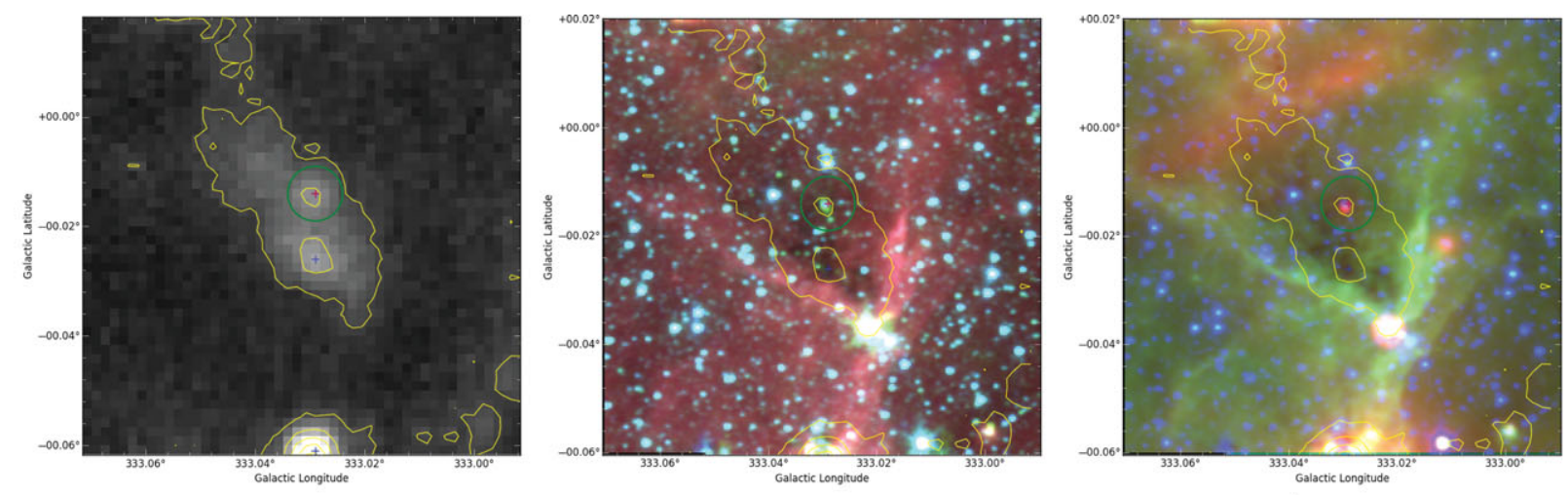

AGAL333.029-00.014_B : $V_{c}=-58.4 \mathrm{~km} \mathrm{~s}^{-1}$
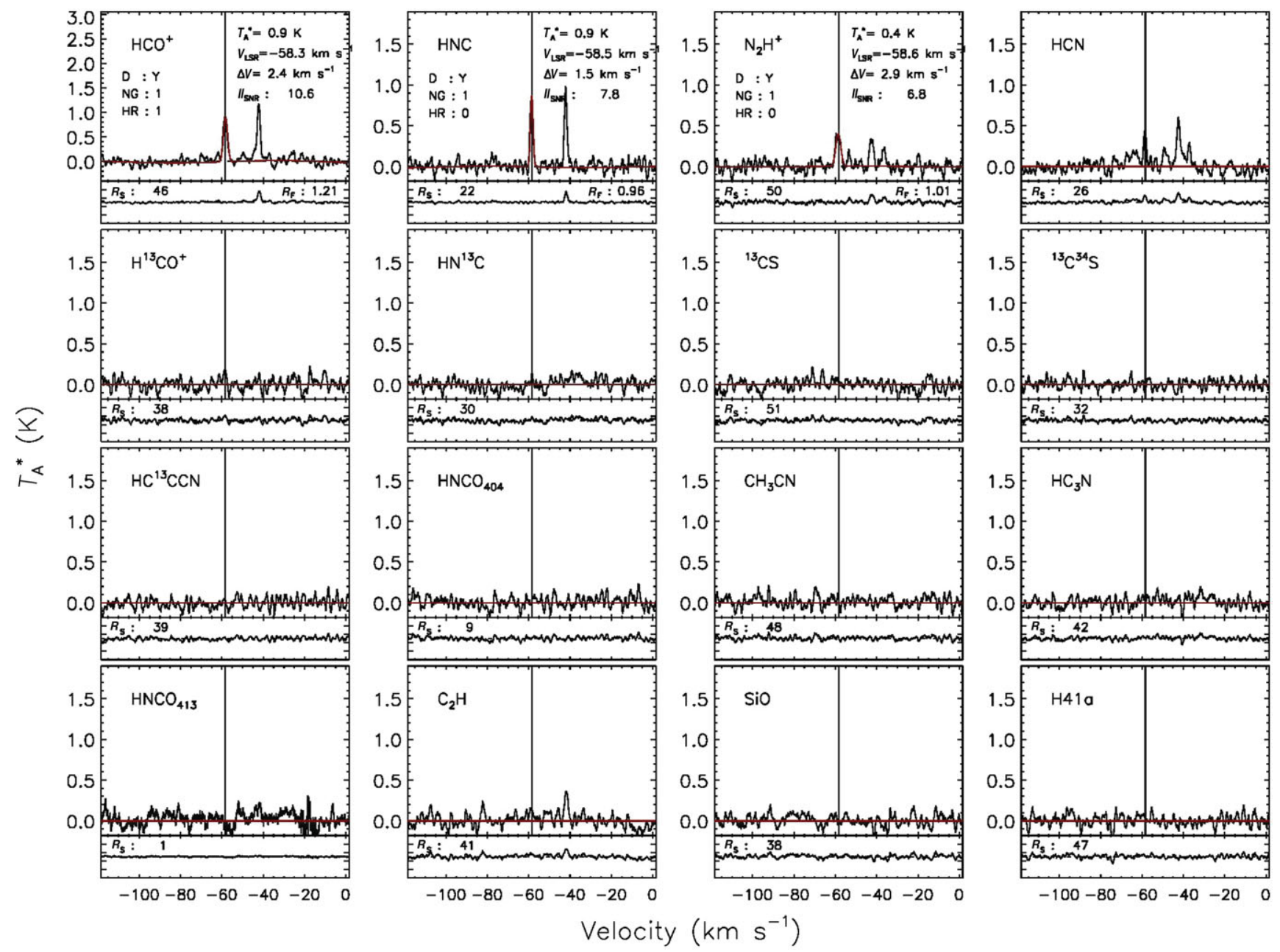

Figure 7. ATLASGAL/Spitzer images and MALT90 spectra towards AGAL333.029-00.014_B : an example of a 'Protostellar' clump with two velocity components detected along the line of sight (component B is shown here, component A is shown in Figure 6). The images and spectra are the same as plotted and described in Figure 1.

emission from the various gas tracers within a clump can often be very different (see e.g., Jackson et al. 2013; Hoq et al. 2013; Stephens et al. 2015). In order to generate a manageable summary of the line emission detected within MALT90, we choose to first catalogue the properties of the line emission towards the dust continuum emission peak of each of the ATLASGAL clumps. This first catalogue will, therefore, not include information on the spatial distribution, velocity field, or line profile variations across the clumps that the maps do provide. Nevertheless, this catalogue provides a summary of the emission detected towards the $870 \mu \mathrm{m}$ dust continuum peak of each clump and an easy 

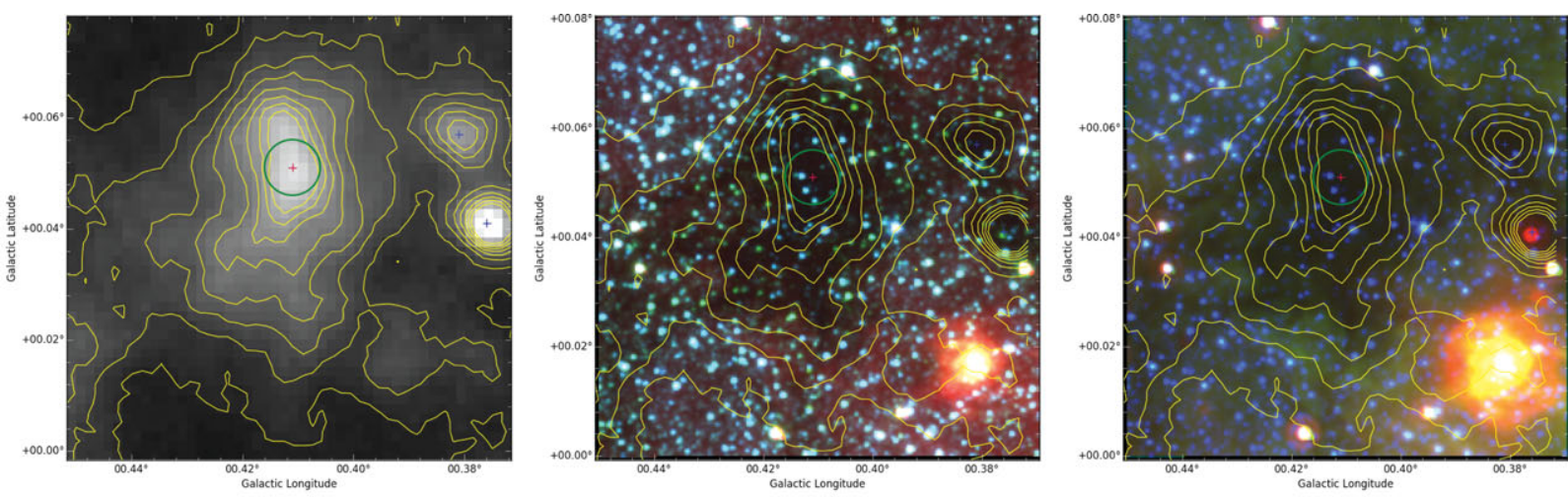

AGAL000.411+00.051_S: $V_{c}=26.4 \mathrm{~km} \mathrm{~s}^{-1}$
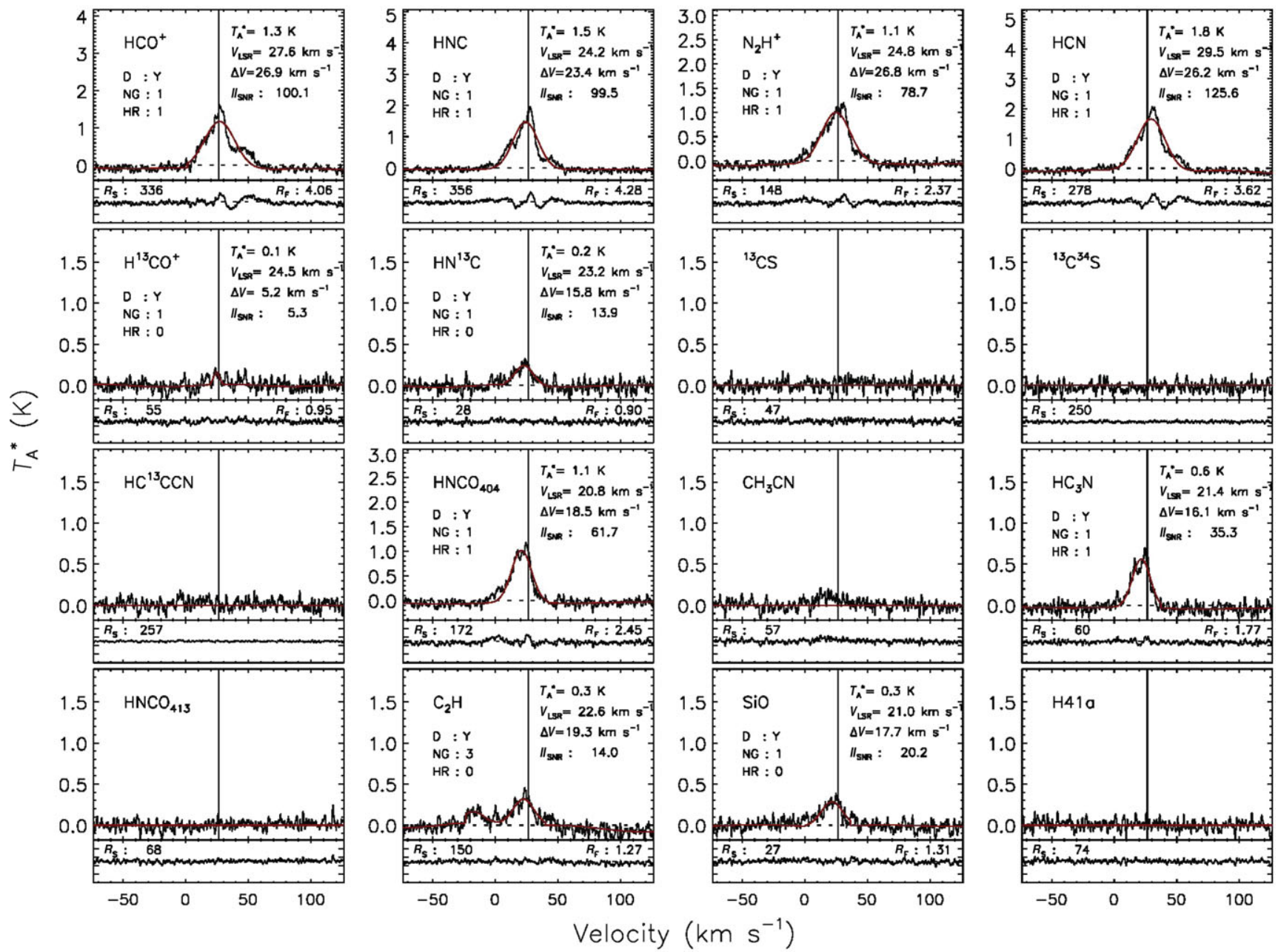

Figure 8. ATLASGAL/Spitzer images and MALT90 spectra towards AGAL000.411+00.051_S: an example of a clump located in the Central Molecular Zone (note the broad line-widths). The images and spectra are the same as plotted and described in Figure 1.

searchable list from which to select clumps for more detailed analyses.

\subsection{Spectra towards each high-mass clump}

For each clump, we have extracted an averaged spectrum from the MALT90 datacubes for each of the 16 emission lines. The spectra were created by averaging the spectra in a $3 \times 3$ pixel $(27$ arcsec $\times 27$ arcsec $)$ box around the clump's dust peak determined from the ATLASGAL catalogues. The size of the averaging box was selected to maximise the signal to noise of the fainter emission and is approximately the angular resolution of MALT90 (for details on the data reduction pipeline, see Jackson et al. 2013). Note that all 
Table 1. Emission lines observed as part of the MALT90 survey.

\begin{tabular}{|c|c|c|c|c|c|}
\hline Emission lines & $\begin{array}{c}\text { Frequency } \\
\quad(\mathrm{GHz})\end{array}$ & $\begin{array}{c}\mathrm{E}_{u} / k^{a} \\
(\mathrm{~K})\end{array}$ & $\begin{array}{c}n_{\text {crit }} \\
\left(\mathrm{cm}^{-3}\right)\end{array}$ & Name & Detections $^{b}$ \\
\hline \multicolumn{6}{|l|}{ Dense, 'low' $\mathrm{E}_{u} / k$ : } \\
\hline $\mathrm{HCO}^{+}(1-0)$ & 89.188526 & 4.28 & $2 \times 10^{5}$ & Formylium & $2874(88.5 \%)$ \\
\hline $\operatorname{HNC}(1-0)$ & 90.663572 & 4.35 & $3 \times 10^{5}$ & Hydrogen isocyanide & $2849(87.8 \%)$ \\
\hline $\mathrm{N}_{2} \mathrm{H}^{+}(1-0)$ & 93.173771 & 4.47 & $3 \times 10^{5}$ & Diazenylium & $2484(76.5 \%)$ \\
\hline $\mathrm{HCN}(1-0)$ & 88.631847 & 4.25 & $3 \times 10^{6}$ & Hydrogen cyanide & $2520(77.6 \%)$ \\
\hline \multicolumn{6}{|c|}{ Dense, 'low' $\mathrm{E}_{u} / k$, optically thin: } \\
\hline $\mathrm{H}^{13} \mathrm{CO}^{+}(1-0)$ & 86.754330 & 4.16 & $2 \times 10^{5}$ & Formylium, isotopologue & $911(28.1 \%)$ \\
\hline $\mathrm{HN}^{13} \mathrm{C}(1-0)$ & 87.090859 & 4.18 & $3 \times 10^{5}$ & Hydrogen isocyanide, isotopologue & $545(16.8 \%)$ \\
\hline${ }^{13} \mathrm{CS}(2-1)$ & 92.494303 & 6.66 & $3 \times 10^{5}$ & Carbon monosulfide, isotopologue & $170(5.2 \%)$ \\
\hline${ }^{13} C^{34} S(2-1)$ & 90.926036 & 7.05 & $4 \times 10^{5}$ & Carbon monosulfide, isotopologue & $8(0.2 \%)$ \\
\hline \multicolumn{6}{|l|}{ Dense, 'high' $\mathrm{E}_{u} / k$ : } \\
\hline $\mathrm{HC}^{13} \mathrm{CCN}(10-9)$ & 90.593059 & 24.37 & $1 \times 10^{6}$ & Cyanoacetylene, isotopologue & $8(0.2 \%)$ \\
\hline HNCO $4(0,4)-3(0,3)$ & 87.925238 & 10.55 & $1 \times 10^{6}$ & Isocyanic Acid & $278(8.6 \%)$ \\
\hline $\mathrm{CH}_{3} \mathrm{CN} 5(1)-4(1)$ & 91.985313 & 20.39 & $4 \times 10^{5}$ & Methyl cyanide & $100(3.1 \%)$ \\
\hline $\mathrm{HC}_{3} \mathrm{~N}(10-9)$ & 90.978989 & 24.01 & $5 \times 10^{5}$ & Cyanoacetylene & $777(23.9 \%)$ \\
\hline HNCO $4(1,3)-3(1,2)$ & 88.239027 & 53.86 & $6 \times 10^{6}$ & Isocyanic acid & $0 \quad(0.0 \%)$ \\
\hline \multicolumn{6}{|l|}{ Dense, 'low' $\mathrm{E}_{\mu} / k$, PDRs: } \\
\hline $\mathrm{C}_{2} \mathrm{H}(1-0) 3 / 2-1 / 2$ & 87.316925 & 4.19 & $4 \times 10^{5}$ & Ethynyl & $1647(50.7 \%)$ \\
\hline \multicolumn{6}{|l|}{ Shocks: } \\
\hline $\mathrm{SiO}(2-1)$ & 86.847010 & 6.25 & $2 \times 10^{6}$ & Silicon monoxide & $319(9.8 \%)$ \\
\hline \multicolumn{6}{|l|}{ Ionised gas: } \\
\hline Н $41 \alpha$ & 92.034475 & & & H-alpha & $20(0.6 \%)$ \\
\hline
\end{tabular}

${ }^{a}$ Excitation energies $\left(\mathrm{E}_{u} / k\right)$, Einstein A coefficients, and collisional rates were obtained from the Leiden atomic and molecular database (LAMDA; Schöier et al. 2005) and Cologne database for molecular spectroscopy (CDMS; Müller et al. 2001, 2005) assuming a gas temperature of $20 \mathrm{~K}$. To derive critical densities, we used calculated collisional rates $(\gamma)$, where possible and the equation $n_{\text {crit }}=A_{\mathrm{u}} / \gamma$, where $A_{\mathrm{u}}$ is the Einstein A coefficient. For many of the more complex species, however, collisional rates have not been calculated. In these cases, we calculate an approximate critical density via $n_{\text {crit }}=A_{\mathrm{u}} /(v \sigma)$, where $v$ is the velocity of the molecule and $\sigma$ is the collisional cross section which was assumed to be $10^{-15} \mathrm{~cm}^{-2}$.

${ }^{b}$ These percentages are calculated with respect to the 3246 ATLASGAL clumps covered by MALT 90.

spectra and Gaussian profile fitting are shown and performed on the $T_{\mathrm{A}}^{*}$ scale. To convert to main-beam brightness temperature $\left(T_{\mathrm{mb}}\right)$, the antenna temperature $\left(T_{\mathrm{A}}^{*}\right)$ should be divided by the main-beam efficiency $\left(\eta_{\mathrm{mb}}\right)$ of 0.49 (Ladd et al. 2005).

Because some MALT90 maps covered more than one ATLASGAL clump, the total number of clumps observed (3 246) is greater than the number of maps obtained (2 014).

\subsection{Additional baseline subtraction}

While a first-order baseline subtraction was performed during the automated processing of the data cubes (see Jackson et al. 2013), the reliability of the Gaussian profile fitting was often considerably improved when we performed an additional higher order fit to the baseline. This was necessary because in poorer weather, the MALT90 spectra were often contaminated by non-linear baselines and baseline ripple, especially at the highest frequency band containing $\mathrm{N}_{2} \mathrm{H}^{+}$. Consequently, a first-order polynomial was often an inadequate model of the baseline, and the automated routine often failed to properly select signal-free velocity ranges. This additional baseline fit and subtraction was individually performed on each spectrum.

\subsection{Noise estimation}

The root-mean-square (rms) noise level in each spectrum was calculated from a comparison of the amplitude for each odd-numbered channel with the amplitude in the preceding even-numbered channel via

$$
\sigma_{\text {noise }}=1.067 \sqrt{\frac{\left\langle\left(y_{2 j+1}-y_{2 j}\right)^{2}\right\rangle_{j}}{2}},
$$

where $y_{j}$ is the amplitude in channel $j$. This assumes Gaussian noise in the presence of a slowly varying spectral signal or baseline variations much broader than the spectral channel spacing. This channel-to-channel estimator of $\sigma_{\text {noise }}$ is robust against the presence of baseline ripples and line emission signals within the spectrum. As such, it represents a more accurate method to reliably determine, in an automated way, the noise level in a spectrum that may have baseline ripples and/or bright line emission anywhere within it, compared to common methods that first require the line to be identified and masked before then applying a simple standard deviation approach. The factor 1.067 corrects for the slight channel-to-channel correlation introduced by the subtraction of a smoothed reference spectrum in the MALT90 data reduction pipeline, the size of this correlation depends on the size of the smoothing window (the pipeline utilised Hanning 


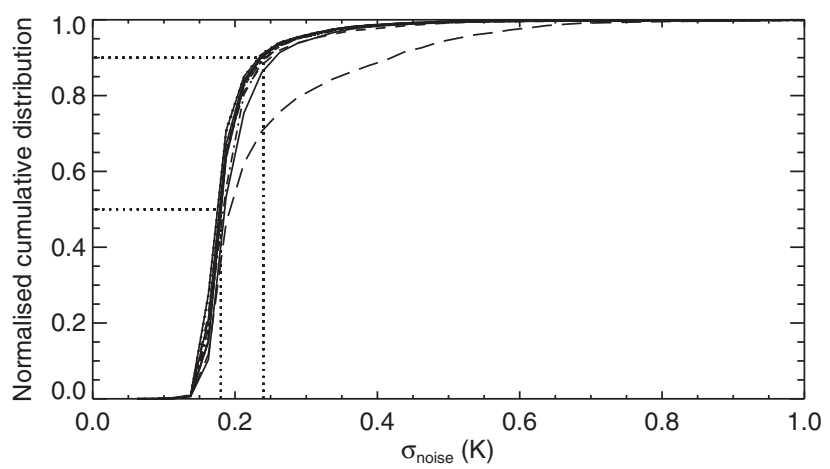

Figure 9. Normalised cumulative distributions of $\sigma_{\text {noise }}$ derived from the spectra of each emission line. The bold line shows the cumulative distribution for the synthetic spectra (see Section 4.5). The tail at high noise levels arises from spectra near the map edges and those obtained when the weather was variable. The noise distribution derived from the spectra of each of the 16 lines follow each other closely, with the exception of HNCO 4(1,3) $3(1,2)$ which suffered from serious band distortions (marked with the dashed line). For all other lines, $90 \%$ of the spectra have $\sigma_{\text {noise }}<0.24 \mathrm{~K}$ and $50 \%$ have $\sigma_{\text {noise }}<0.18 \mathrm{~K}$ (these $\sigma_{\text {noise }}$ levels are marked with the dotted lines).

smoothing with an 11-channel window, see Jackson et al. 2013).

Figure 9 shows the normalised cumulative distribution of $\sigma_{\text {noise }}$ derived from the spectra of each emission line. The bold line shows the cumulative distribution for the synthetic spectra (see Section 4.5). The tail at high noise levels arises from spectra near the map edges and those obtained when the weather was marginal for $90 \mathrm{GHz}$ observations. The derived noise distribution from the 16 emission lines follow each other closely, with the exception of HNCO 4(1,3)$3(1,2)$ which suffered from serious noise spikes and/or band distortions (shown with the dashed line). We examined all possible 'detections' of HNCO 4(1,3)-3(1,2) and concluded that there were no credible signals in any of the spectra; the detection flag in the catalogue is always set to ' $N$ ' for this emission (see Section 2.4.3). For all other emission lines, $90 \%$ of the spectra have $\sigma_{\text {noise }}<0.24 \mathrm{~K}$, while $50 \%$ have $\sigma_{\text {noise }}<0.18 \mathrm{~K}$ on the antenna temperature $\left(T_{\mathrm{A}}^{*}\right)$ scale.

\subsection{Gaussian profile fitting}

In order to characterise the detected emission towards each clump, we have fit each spectrum with either a single or multiple Gaussian profile(s). These profile fits provide key parameters typically used to describe line emission: peak antenna temperature $\left(T_{\mathrm{A}}^{*}\right), \mathrm{FWHM}(\Delta V)$, and central velocity $\left(V_{\mathrm{LSR}}\right)$. In the catalogue, we report the integrated intensity (II) calculated directly from summing $T_{\mathrm{A}}^{*}$ over all channels in the fit range (each channel is independent, with a spectral resolution of $\mathrm{d} v$ ). We choose to calculate II by summing the actual emission, rather than using the derived Gaussian parameters, since the former will also include any component of the line that is non-Gaussian (the exception to this is for $\mathrm{C}_{2} \mathrm{H}$; in this case, we sum under the Gaussian profile fit, since the hyperfine components are well-separated in velocity). The II signal-to-noise ratio, $\mathrm{II}_{\mathrm{SNR}}$, was determined by dividing the II by $\sigma_{I I}$, where $\sigma_{I I}=\sqrt{N_{\text {channel }}} \sigma_{\text {noise }} \mathrm{d} v$, and $N_{\text {channel }}$ is the number of channels in the fitting region. The antenna temperature signal-to-noise ratio, $T_{\mathrm{SNR}}$, was defined as $T_{\mathrm{SNR}}=T_{\mathrm{A}}^{*} / \sigma_{\text {noise }}$.

The profile fitting was performed on the baselinesubtracted spectra as a two-step process: the first iteration was performed on the most prominent peaks in the $\mathrm{HCO}^{+}$, $\mathrm{HNC}$, and $\mathrm{N}_{2} \mathrm{H}^{+}$spectra to determine a 'consensus' velocity $\left(V_{\mathrm{C}}\right)$, while the second iteration was performed on all 16 lines restricting the velocity range over which to search for emission to be $V_{\mathrm{C}} \pm 5 \mathrm{~km} \mathrm{~s}^{-1}$. This two-step method was employed as it resulted in significantly higher detection rates and more reliable fits to the fainter emission.

\subsubsection{Determining a consensus velocity}

For each clump, we determine a single $V_{\mathrm{C}}$ to describe its associated line emission. Given that the transitions covered by the MALT90 survey probe different physical and chemical conditions, there may be slight differences in the derived $V_{\text {LSR }}$ and specific kinematic signatures only evident from certain species (e.g., shocks and outflows from $\mathrm{SiO}$ and $\mathrm{HCO}^{+}$). While these differences are informative, to simplify and summarise the derived velocities from the ensemble of molecular transitions, we determine and report a single $V_{\mathrm{C}}$ which is an estimator of the systemic velocity of the clump. Determining a single $V_{\mathrm{C}}$ for each clump is important, since it will be used to derive the clump's kinematic distance (Whitaker et al. in preparation) and in combination with the dust continuum emission (Guzmán et al. 2015), their masses and luminosities (Contreras et al. in preparation) .

To derive a $V_{\mathrm{C}}$ for the clumps, we first focus on fitting the most commonly detected and brightest emission lines covered by the survey: $\mathrm{HCO}^{+}, \mathrm{HNC}$, and $\mathrm{N}_{2} \mathrm{H}^{+}$. In determining the $V_{\mathrm{C}}$, we the bright $\mathrm{HCN}$ transition because the combination of high optical depths, hyperfine structure, and deep self-absorption often seen in this line resulted in complicated, non-Gaussian profiles, which led to unreliable velocities.

For each clump, the channel with the highest intensity within the $\mathrm{HCO}^{+}, \mathrm{HNC}$, and $\mathrm{N}_{2} \mathrm{H}^{+}$spectra were first identified. A profile was then fit to the spectrum using this brightest channel as the initial guess for the source velocity. For $\mathrm{HCO}^{+}$and $\mathrm{HNC}$, the profile was a single Gaussian; for $\mathrm{N}_{2} \mathrm{H}^{+}$, the profile was a triple Gaussian constrained in velocity using the known hyperfine velocity offsets and a single line-width. If the fit produced a 'reliable' detection (i.e., with an $\mathrm{II}_{\mathrm{SNR}}>4$ and $T_{\mathrm{SNR}}>1$ ), then the velocity and $T_{\mathrm{A}}^{*}$ for this profile were recorded, and the profile was subtracted from the spectrum. A second profile was then fit to the next brightest peak in the spectrum and was recorded if it too was a reliable detection. This resulted in, at most, six velocities: two distinct velocity components each for the $\mathrm{HNC}, \mathrm{HCO}^{+}$, and $\mathrm{N}_{2} \mathrm{H}^{+}$.

The velocities were sorted by increasing value and aggregated in a single pass: starting from the lowest velocity, if the 
next higher velocity was within $5 \mathrm{~km} \mathrm{~s}^{-1}$, then the two velocities were averaged, weighted by their amplitudes. After this procedure, the component with the greatest accumulated weight was identified as the first velocity component. If more than one velocity component survived the averaging procedure (i.e., their separation in velocity was $>5 \mathrm{~km} \mathrm{~s}^{-1}$ ), the component with the next highest weight was designated as the second velocity component.

For spectra where no second component was identified, we report in the catalogue a single velocity component towards that dust peak and append an ' $S$ ' (for 'single') to the clump's name. In cases where two velocity components were identified and their velocities were within $15 \mathrm{~km} \mathrm{~s}^{-1}$ of each other, we consider the emission to arise from the same physical clump (15 $\mathrm{km} \mathrm{s}^{-1}$ was used to separate velocity components since this was three times the typically derived value for the line-width, $\sim 5 \mathrm{~km} \mathrm{~s}^{-1}$ from $\mathrm{HCO}^{+}$). We report a single velocity component towards that dust peak (clump name in the catalogue is appended with an ' $S$ ') but indicate this decision by setting the number of Gaussians flag, ' $N G$ ', in the catalogue to 2 (i.e., two Gaussian profiles were combined). In these cases, we report in the catalogue values for $T_{\mathrm{A}}^{*}, V_{\mathrm{LSR}}$, and $\Delta V$ (and their uncertainties) derived from a moment (intensity weighted) calculation of the combined line profile.

If the velocities from the two components were $>15 \mathrm{~km} \mathrm{~s}^{-1}$ apart, we consider the emission to arise from physically separate clumps along the line of sight and thus report two velocity components towards that dust peak (clump names in the catalogue are appended with an 'A' and ' $\mathrm{B}$ '). In these cases, the first velocity component with the greatest sum in $T_{\mathrm{A}}^{*}$ derived from the three lines is reported as component ' $A$ ', the second velocity component is reported as component ' $\mathrm{B}$ '.

A $V_{\mathrm{C}}$ was determined for $\sim 94 \%$ of our clumps; typically all three lines $\left(\mathrm{HNC}, \mathrm{HCO}^{+}\right.$, and $\mathrm{N}_{2} \mathrm{H}^{+}$) were detected, and their velocities agreed within $<1 \mathrm{~km} \mathrm{~s}^{-1}$. A second velocity component was identified in $19 \%$ of the spectra; in $78 \%$ of these cases, the additional velocity component appeared in only a single molecular line.

\subsubsection{Fits to individual spectra}

Once the $V_{\mathrm{C}}$ for each clump was determined, a Gaussian profile was then fit to all 16 spectra by setting the search region to be $V_{\mathrm{C}} \pm 5 \mathrm{~km} \mathrm{~s}^{-1}$. If a $V_{\mathrm{C}}$ was not found (i.e., there was no significant emission from $\mathrm{HCO}^{+}, \mathrm{HNC}$, or $\mathrm{N}_{2} \mathrm{H}^{+}$), then the search region was left unconstrained in velocity.

To increase the reliability of finding emission from the fainter lines at the same velocity as the brighter lines, the velocity range over which the fitting was performed was also restricted. The fitting range was determined individually for each spectrum: its centre was defined using the channel of the peak intensity found within the search region. The fitting range was then expanded in both positive and negative velocity directions until the first negative channel was found (i.e., the noise level was reached). As an extra buffer and to ensure that the fitting range was both wide enough to cover the emission and to include a sufficient number of channels to accurately describe the baseline, the fitting range was then increased by an additional 25 channels in both the positive and negative velocity directions $\left(2.75 \mathrm{~km} \mathrm{~s}^{-1}\right)$ to establish the final fitting range.

Gaussian profiles were then fit to the spectrum within the fitting range. For the brightest velocity component (either ' $S$ ' or 'A'), the first fit performed was that using a single Gaussian profile. A second fit was then performed using multiple Gaussian profiles. For molecules showing hyperfine splitting $\left(\mathrm{C}_{2} \mathrm{H}, \mathrm{HCN}\right.$, and $\left.\mathrm{N}_{2} \mathrm{H}^{+}\right)$, the second fit used multiple Gaussian components (either 2, 3, or 3, respectively) constrained in velocity using the known hyperfine velocity offsets for each molecule, a single line-width, but unconstrained amplitudes. ${ }^{2}$ For all other molecules, the second fit was performed using two unconstrained Gaussian profiles.

When a second velocity component was evident in the spectra (i.e., for components 'B'), we only perform a fit using a single Gaussian profile. The fitting for these components was simplified as they were often faint and rarely showed a profile that was not well fit by a simple, single Gaussian profile.

\subsubsection{Selecting the best fit}

For each fit, a $\chi^{2}$ minimisation was performed to determine the best-fitting parameters, their uncertainties, and a fit probability $\left(T_{\mathrm{A}}^{*}, \Delta V, V_{\mathrm{LSR}}, \mathrm{II}\right.$, and $\left.P_{\mathrm{fit}}\right)$. The fit probability is the integral of the normalised $\chi^{2} /$ DoF distribution above the value determined by the fit (where DoF is the number of fit degrees of freedom). It, therefore, represents the probability that in an ensemble of well-modelled observations, a value of $\chi^{2} /$ DoF greater than the observed value would arise due to statistical fluctuations. We found that the distributions of fit probabilities were reasonably flat, as expected if the Gaussian profile is appropriate to the line shape and the noise is accurately represented. A spike in the distribution at very small fit probability indicates cases where the fit was a poor representation of the data and its uncertainties.

We compared the probabilities of the two fits (either the single Gaussian profile fit or the multiple Gaussian profile fit) to select which line parameters to report. For lines with hyperfine structure, we gave preference to the multipleconstrained-Gaussian fit over the single-Gaussian fit, for the other lines, we gave preference to the single-Gaussian fit over the double unconstrained Gaussian fit. We report in the catalogue parameters from the 'preferred' fit when its probability was $>1 \%$. If this probability was $<1 \%$, then the 'non-preferred' fitting parameters were reported, but only

\footnotetext{
${ }^{2}$ While the $\mathrm{N}_{2} \mathrm{H}^{+}(1-0)$ hyperfine splitting will comprise 15 individual components, the typical line-widths measured towards these clumps is broader than the width of the blended hyperfine components. As such, they will combine to blend into three spectral features. Extensive testing of our fitting algorithm revealed that fitting a simplified three-component Gaussian profile was more than sufficient to characterise these data.
} 
if they had a fit probability greater than the probability of the preferred fit. If this was not the case, then we report the preferred fit, regardless of its fit probability.

Included in the catalogue is a detection flag ('D') to indicate the significance of the detection: it is set to ' $\mathrm{Y}$ ' when the line is well detected $\left(T_{\mathrm{SNR}}>1\right.$ and $\left.\mathrm{II}_{\mathrm{SNR}}>4.0\right)$, ' $\mathrm{M}$ ' when the detection is marginal $\left(T_{\mathrm{SNR}}>1\right.$ and $3.0<\mathrm{II}_{\mathrm{SNR}}<$ $4.0)$, and ' $\mathrm{N}$ ' when there is no reliable detection $\left(T_{\mathrm{SNR}}<\right.$ 1 or $\mathrm{II}_{\mathrm{SNR}}<3.0$ ). We indicate the number of Gaussians in the selected fit via the flag, 'NG'; the values correspond to: 1 for a single Gaussian profile fit, 2 for the two-component unconstrained Gaussian profile fit, 3 for a fit that includes the hyperfine structure, and -1 when no good fit was determined. When the best fit determined was the two-component unconstrained Gaussian profile (' $\mathrm{NG}$ ' $=2$ ), we report in the catalogue values for $T_{\mathrm{A}}^{*}, V_{\mathrm{LSR}}$, and $\Delta V$ (and their uncertainties) derived from a moment (intensity weighted) calculation of the combined line profiles. In many cases, these spectra show clear self-absorption and evidence for outflow/infall motions.

\subsubsection{Spectrum residuals}

With the best-fitting profile selected and subtracted, we then determined the residuals $\left(T-T_{\text {fit }}\right)$ across both the fit range $\left(R_{\mathrm{f}}\right)$ and in the complete spectrum $\left(R_{\mathrm{s}}\right)$. The parameter $R_{\mathrm{f}}$ reports the ratio in the standard deviation in the residual spectrum in the fit range to the standard deviation derived from a signal-free portion of the spectrum. Visual inspection of the spectra showed that values for $R_{\mathrm{f}} \gtrsim 1.5$ indicate cases where there is significant emission or absorption remaining in the fit range after the best-fitting profile has been removed. As such, we use a value for $R_{\mathrm{f}}$ of $\gtrsim 1.5$ to designate those spectra with 'high residuals (HR)'. This parameter helps identify spectra that show non-Gaussian profiles indicative of self-absorption or infall/outflow motions. We indicate cases where $R_{\mathrm{f}}>1.5$ in the catalogue by setting the HR flag to 1 .

The second parameter, $R_{\mathrm{s}}$, reports the number of channels in the residual spectrum that are $>3 \sigma_{\text {noise }}$ after removal of all best-fitting profiles. This parameter is useful for identifying spectra that contain other velocity components within the spectrum that were missed by the automated component selection and fitting routine. A high value of $R_{\mathrm{s}}$ indicates either very broad lines or more than two velocity components. High values for this parameter (i.e., >70) are typically seen towards clumps in the Galactic centre where the emission is complex and not well fit by Gaussian profile(s).

\subsubsection{Opacities}

For all clumps in which the $\mathrm{N}_{2} \mathrm{H}^{+}$emission could be fit with a three component Gaussian profile (i.e., in 2256 cases), we have derived an optical depth $(\tau)$ of the main hyperfine component $\left(J^{\prime} F_{1}^{\prime} F^{\prime} \rightarrow J F_{1} F=123 \rightarrow 012\right)$. Assuming LTE, where the excitation temperatures of the three components are the same, we follow the description outlined in Shirley et al. (2005) and calculate the optical depth of the main hyperfine component by taking the ratios of the ob- served antenna temperatures of the left and right components to the main component (i.e., the 101-012, 112-012, and 123012 hyperfine components, respectively; these are labelled by their $J^{\prime} F_{1}^{\prime} F^{\prime} \rightarrow J F_{1} F$ transitions). The optical depth was determined numerically by finding the minimum of the function:

$f(\tau)=\left(2-\left(\frac{T_{\mathrm{L}}\left(1-e^{-\tau}\right)}{T_{\mathrm{M}}\left(1-e^{-r_{\mathrm{L}} \tau}\right)}\right)^{2}-\left(\frac{T_{\mathrm{R}}\left(1-e^{-\tau}\right)}{T_{\mathrm{M}}\left(1-e^{-r_{\mathrm{R}} \tau}\right)}\right)^{2}\right)^{2}$

over the range $-3<\tau<6$, where $T_{\mathrm{L}}, T_{\mathrm{M}}$, and $T_{\mathrm{R}}$ are the fitted $T_{\mathrm{A}}^{*}$ amplitudes for the left, main, and right components, respectively and $r_{L}$ and $r_{R}$ are the relative intensities calculated based on each clump's line width (see Shirley et al. 2005 for details).

\subsection{Completeness and reliability analysis}

To determine the completeness and reliability of the detections reported in the catalogue, we generated a series of synthetic spectra which were passed through the same automated line-fitting procedure as described above. For this analysis, we assume that there is a single Gaussian component in each spectrum (i.e., that there is one velocity component along the line of sight), that all profiles are Gaussian, and that the simulated emission arises from a molecular transition that shows no hyperfine splitting. All values for, or derived from, the synthetic spectra have the subscript 'syn'.

The synthetic spectra were based on noise spectra drawn randomly from the MALT90 maps for $\mathrm{HC}^{13} \mathrm{CCN},{ }^{13} \mathrm{C}^{34} \mathrm{~S}$, and $\mathrm{H} 41 \alpha$ (these maps were selected since the emission from these transitions were rarely detected). A candidate noise spectrum was generated by averaging spectra in a $3 \times 3$ pixel box selected randomly according to the distribution of ATLASGAL clump peak positions within the MALT90 maps. The noise spectrum was passed through the baseline subtraction and Gaussian fitting procedure; if a signal was detected with peak amplitude greater than 1.5 times the $\sigma_{\text {noise }}$ of the spectrum then that noise spectrum was rejected. Amplitudes of an accepted candidate noise spectrum were then multiplied by -1.0 to produce a final noise spectrum for use in the analysis. The output of this process was an ensemble of 10000 noise spectra that statistically sampled the maps with the same weighting in relevant parameters (e.g., observing conditions, galactic longitude, and position within the maps) as the spectra extracted towards each clump.

\subsubsection{Completeness}

To determine the completeness of the reported detections, we superposed Gaussian profiles on the noise spectra and then passed these synthetic spectra through the automated linefitting procedure. Values for the peak antenna temperature $\left(T_{\text {syn }}\right)$ were selected uniformly from 0.0 to $1.0 \mathrm{~K}$; values for the line-width $\left(\Delta V_{\text {syn }}\right)$ were selected uniformly from 1.6 to $5 \mathrm{~km} \mathrm{~s}^{-1}$; and values for the central velocity $\left(V_{\text {syn }}\right)$ were selected randomly from the range $\pm 150 \mathrm{~km} \mathrm{~s}^{-1}$. The $V_{\mathrm{C}}$ was 
set randomly in the range $\pm 3 \mathrm{~km} \mathrm{~s}^{-1}$ around $V_{\text {syn }}$. These were chosen as they represent the typical values and ranges for these parameters measured within the survey data. We use the same detection criteria for the synthetic spectra as we applied to the real data: a synthetic profile was considered to be 'detected' if the $\mathrm{II}_{\mathrm{SNR}}$ was $>4$, and the $T_{\mathrm{SNR}}>1$. We impose one additional criterion for the synthetic spectra: the derived central velocity from the Gaussian profile fit must be within $\pm 1 \mathrm{~km} \mathrm{~s}^{-1}$ of the input $V_{\text {syn }}$.

We used the synthetic spectra to determine the probability of detecting a line (completeness) as a function of our detection criteria. The upper panel of Figure 10 shows completeness as a function of $T_{\mathrm{SNR} \text {,syn }}$ (i.e., $T_{\text {syn }} / \sigma_{\text {noise }}$ ); the middle panel shows completeness as a function of the ratio of $\mathrm{II}_{\mathrm{SNR} \text {,syn }}$ (i.e., $\mathrm{II}_{\text {syn }} / \sigma_{\text {noise }}$ ). In both panels, the dashed curve corresponds to the expanded scale shown on the right. The dotted vertical lines mark a $T_{\mathrm{SNR}, \text { syn }}$ of 1 and an $\mathrm{II}_{\mathrm{SNR}, \text { syn }}$ of 3 and 4, which correspond to the criteria imposed for detections to be included within the catalogue.

We find that the completeness versus $T_{\mathrm{SNR} \text {,syn }}$ rises rapidly, a consequence of the peak-fitting algorithm starting from the highest amplitude channel in the search region. Completeness versus $\mathrm{II}_{\mathrm{SNR}, \text { syn }}$ rises somewhat more slowly, reaching $80 \%$ by $\mathrm{II}_{\mathrm{SNR} \text {,syn }}=4$ and $99 \%$ by $\mathrm{II}_{\mathrm{SNR} \text {,syn }}=8$. The lower panel of Figure 10 shows the completeness as a function of $T_{\text {syn }}$. Since $T_{\text {syn }}$ in these spectra represents the measured $T_{\mathrm{A}}^{*}$ from our data, these results indicate that we achieve a completeness of $>95 \%$ for detections with a measured $T_{\mathrm{A}}^{*}>$ $0.4 \mathrm{~K}$ and $>99 \%$ for detections with a $T_{\mathrm{A}}^{*}>0.6 \mathrm{~K}$.

\subsubsection{Reliability}

Passing the noise spectra through our standard analysis procedure provided a measure of the reliability of detections, i.e., the probability of an accidental (false-positive) detection, as a function of detection criteria. Figure 11 shows the normalised cumulative distribution in $\mathrm{II}_{\mathrm{SNR}}$ determined from the noise spectra (note, this will characterise accidental detections due to noise and baseline fluctuations). The probability of a false-positive detection is $<0.3 \%$ for an $\mathrm{II}_{\mathrm{SNR}}>3$.

By comparing the fitting results with the known characteristics used to generate the synthetic profiles, we have also determined the reliability with which the fitting procedure recovers the Gaussian parameters: amplitude, velocity, linewidth, and II, and the accuracy with which it estimates their uncertainties. Figure 12 compares the derived parameters to the input values (panels are amplitude, line-width, velocity, and II, respectively, from upper to lower). For the amplitude, line-width, and II, we calculate the ratio of derived values to input values; for the velocity, we calculate the difference. For $\operatorname{input} T_{\text {syn }}>0.24 \mathrm{~K}$, the distributions are close to unity, which indicates that the fitting procedure accurately determines all three Gaussian parameters (amplitude, line width, and II) and introduces no significant bias in its estimations. Indeed, the relative uncertainty in these quantities, as estimated by the standard deviations of the ratios from unity (shown as
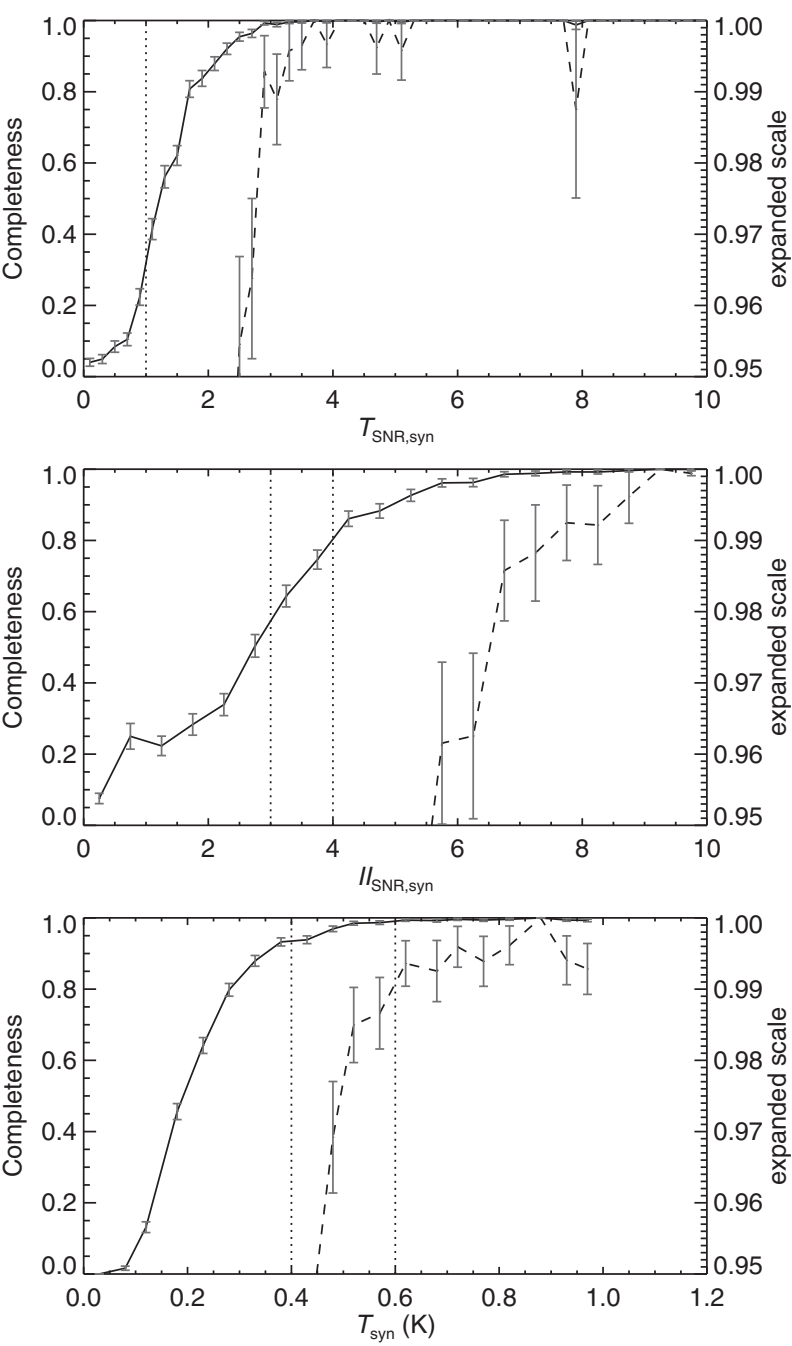

Figure 10. Completeness (probability of detecting a line) as a function of the $T_{\mathrm{SNR}, \text { syn }}$ (upper panel), $\mathrm{II}_{\mathrm{SNR}, \text { syn }}$ (middle panel), and input $T_{\text {syn }}$ (lower panel). In all panels, the dashed curves correspond to the expanded scale shown on the right axis. The error bars show the statistical uncertainties in the synthetic sample. The vertical dotted lines in the upper and middle panels mark the selection criteria imposed for detections to be included within the catalogue (i.e., $T_{\mathrm{SNR}}>1$ and $\mathrm{II}_{\mathrm{SNR}}>3$ or $>4$, for 'marginal' and 'reliable' detections, respectively). The vertical dotted lines in the lower panel mark the completeness levels. The achieved completeness levels are $>95 \%$ for detections with a measured $T_{\mathrm{A}}^{*}>0.4 \mathrm{~K}$ and $>99 \%$ for detections with a $T_{\mathrm{A}}^{*}>0.6 \mathrm{~K}$.

the error bars), was $\sim 20 \%$ for $T_{\text {syn }} \sim 0.24 \mathrm{~K}$ and improved to $<5 \%$ for $T_{\text {syn }}>1 \mathrm{~K}$.

\section{DESCRIPTION OF THE CATALOGUE}

The catalogue contains an entry for each of the clumps covered by the MALT90 survey. While the total number of spectra extracted towards the clumps is 3246 , in 311 cases there were two distinct velocity components detected along the line of sight. In these cases, we report separate entries ('A' and 'B' components) which leds to a total of 3556 entries in the catalogue. 


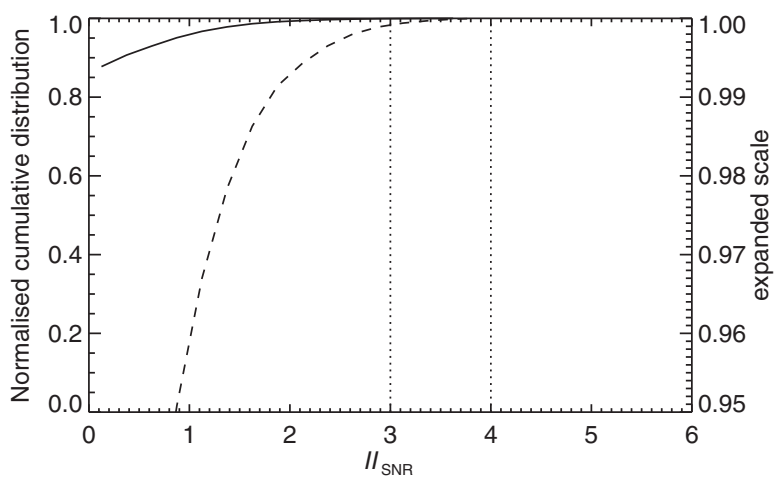

Figure 11. Normalised cumulative distributions in $\mathrm{II}_{\mathrm{SNR}}$ for the synthetic noise spectra (note, this will characterise accidental detections due to noise and baseline fluctuations). The dashed curves correspond to the expanded scale shown on the right axis. The vertical dotted lines mark the selection criteria imposed for detections to be included within the catalogue: an $\mathrm{II}_{\mathrm{SNR}}$ of $>3$ for 'marginal' and $>4$ for 'reliable' detections, respectively. We find that the probability of a false-positive detection is $<0.3 \%$ for an $\mathrm{II}_{\mathrm{SNR}}>3$.

Table 2 gives a sample of a subset of the catalogue entries. For brevity, we include here, as an example, a description of the derived properties for $\mathrm{HCO}^{+}(1-0)$. The full catalogue contains the same entries (i.e., columns 10-25) repeated for each of the 16 transitions covered by the survey (available via the aforementioned data link).

The columns of Table 2 are as follows:

(1) The clump name [from the ATLASGAL catalogues (Contreras et al. 2013 and Urquhart et al. 2014a)], appended with an 'S', 'A', or 'B'; (2) and (3) the Galactic coordinates of the clumps' ATLASGAL $870 \mu \mathrm{m}$ emission peak $(\ell, b$, in degrees); (4) the ATLASGAL $870 \mu \mathrm{m}$ peak flux (in Jy); (5) the IR-based category assigned to the clump (S), 'Q' for quiescent, 'A' for protostellar, 'C' for compact $\mathrm{H}$ II regions, ' $\mathrm{H}$ ' for extended $\mathrm{H}_{\text {II }}$ regions, ' $\mathrm{P}$ ' for photo-dissociation regions, or ' $U$ ' for uncertain; (6) the consensus velocity $\left(V_{\mathrm{C}}\right.$ in $\mathrm{km} \mathrm{s}^{-1}$ ) determined as the intensity weighted velocity derived from reliable fits to $\mathrm{HCO}^{+}, \mathrm{HCN}$, and $\mathrm{N}_{2} \mathrm{H}^{+}$; (7) the total number of lines detected towards this clump with $\mathrm{II}_{\mathrm{SNR}}>3.0(\mathrm{NL}) ;(8)$ and $(9)$ the measured optical depth $(\tau)$ and its uncertainty; (10) the flag indicating whether emission was detected (D), ' $\mathrm{Y}$ ' when the line is well detected $\left(\mathrm{II}_{\mathrm{SNR}}>\right.$ 4.0), 'M' when the detection is marginal $\left(3.0<\mathrm{II}_{\mathrm{SNR}}<\right.$ 4.0), and ' $\mathrm{N}$ ' when there is no reliable detection; (11) the flag indicating the number of Gaussian profiles that were fit (NG), 1 for a single Gaussian profile fit, 2 for the twocomponent unconstrained Gaussian profile fit, 3 for a fit that includes the hyperfine structure, and -1 when no good fit was determined; (12) and (13) the derived antenna temperature and its uncertainty from the Gaussian fitting $\left(T_{\mathrm{A}}^{*}\right.$, in $\left.\mathrm{K}\right)$; (14) and (15) the derived velocity and its uncertainty from the Gaussian fitting $\left(V_{\mathrm{LSR}}\right.$, in $\left.\mathrm{km} \mathrm{s}^{-1}\right)$; (16) and (17) the derived velocity FWHM and its uncertainty from the Gaussian fitting $\left(\Delta \mathrm{V}\right.$, in $\left.\mathrm{km} \mathrm{s}^{-1}\right)$; (18) and (19) the II (in $\left.\mathrm{K} \mathrm{km} \mathrm{s}^{-1}\right)$ and its uncertainty; (20) the $1 \sigma \mathrm{rms}$ noise of the spectrum $\left(\sigma_{\text {noise }}\right.$ in $\left.\mathrm{km} \mathrm{s}^{-1}\right) ;(21)$ the fit probability $\left(\mathrm{P}_{\mathrm{fit}}\right) ;(22)$ the $\mathrm{II}_{\mathrm{SNR}} ;(23)$

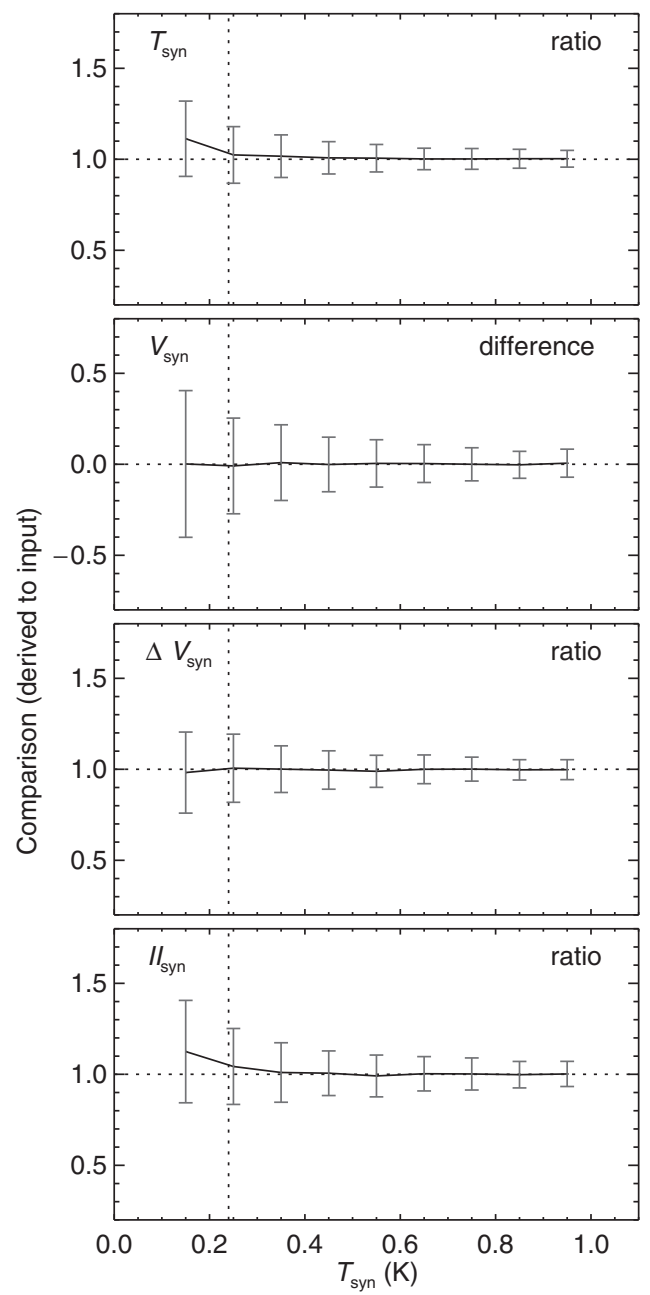

Figure 12. Reliability with which the derived parameters (amplitude, velocity, line-width, and integrated intensity, upper to lower panels, respectively) are estimated. These reliabilities were determined by comparing the fitting results with the known characteristics used to generate the synthetic profiles (these comparisons are shown as ratios, with the exception of velocity, which are differences). The error bars reflect the uncertainty with which the automated routine estimates their values. The dotted vertical lines mark a $T_{\text {syn }}$ of $0.24 \mathrm{~K}$ ( $90 \%$ of the spectra have noise less than this value).

the ratio in the standard deviation in the residual spectrum in the fit range to that determined from a signal-free portion of the spectrum $\left(\mathrm{R}_{f}\right)$; (24) the flag indicating if the profile has a HR in the fit range, $\mathrm{R}_{f}>1.5(\mathrm{HR})$; and (25) the total number of channels in the residual spectrum that are $>3 \sigma$ after removal of all best-fitting profiles $\left(R_{\mathrm{s}}\right)$.

\section{EXAMPLES: IMAGES AND SPECTRA}

Figures 1-8 provide an overview of the ATLASGAL/Spitzer images and MALT90 spectra towards a selection of clumps that are contained within the catalogue. We show here clumps that are representative of those in each of the IR-based categories: Figure 1 shows a quiescent clump, Figure 2 a protostellar clump, Figures 3 and $4 \mathrm{H}_{\text {II }}$ regions, 
Table 2. An extract of the line emission towards the high-mass clumps covered by MALT90. See Section 5 for a detailed description of its contents.

\begin{tabular}{|c|c|c|c|c|c|c|c|c|c|c|c|c|c|c|c|c|c|c|c|c|c|c|c|c|}
\hline \multirow[b]{2}{*}{$\begin{array}{l}\text { Clump name } \\
\text { (1) }\end{array}$} & \multicolumn{3}{|c|}{ Dust peak } & \multirow[b]{2}{*}{$\begin{array}{c}S \\
(5)\end{array}$} & \multirow[b]{2}{*}{$\begin{array}{l}V_{\mathrm{C}} \\
\text { (6) }\end{array}$} & \multirow[b]{2}{*}{$\begin{array}{l}\mathrm{NL} \\
\text { (7) }\end{array}$} & \multirow[b]{2}{*}{$\begin{array}{c}\tau \\
(8)\end{array}$} & \multirow[b]{2}{*}{$\begin{array}{l}\tau_{\mathrm{err}} \\
(9)\end{array}$} & \multicolumn{16}{|c|}{$\mathrm{HCO}^{+}(1-0)$} \\
\hline & $\begin{array}{c}\ell \\
\text { (2) }\end{array}$ & $\begin{array}{c}b \\
(3)\end{array}$ & $\begin{array}{l}\text { flux } \\
\text { (4) }\end{array}$ & & & & & & $\begin{array}{c}\mathrm{D} \\
(10)\end{array}$ & $\begin{array}{l}\text { NG } \\
(11)\end{array}$ & $\begin{array}{c}T_{\mathrm{A}}^{*} \\
(12)\end{array}$ & $\begin{array}{l}T_{\text {Aerr }}^{*} \\
(13)\end{array}$ & $\begin{array}{l}V_{\mathrm{LSR}} \\
(14)\end{array}$ & $\begin{array}{l}V_{\text {err }} \\
(15)\end{array}$ & $\begin{array}{c}\Delta V \\
(16)\end{array}$ & $\begin{array}{l}\Delta V_{\text {err }} \\
(17)\end{array}$ & $\begin{array}{c}\text { II } \\
(18)\end{array}$ & $\begin{array}{l}\mathrm{II}_{\text {err }} \\
(19)\end{array}$ & $\begin{array}{l}\sigma_{\text {noise }} \\
(20)\end{array}$ & $\begin{array}{l}P_{\text {fit }} \\
(21)\end{array}$ & $\begin{array}{c}\mathrm{II}_{\mathrm{SNR}} \\
(22)\end{array}$ & $\begin{array}{c}R_{\mathrm{f}} \\
(23)\end{array}$ & $\begin{array}{l}\text { HR } \\
(24)\end{array}$ & $\begin{array}{c}R_{\mathrm{S}} \\
(25)\end{array}$ \\
\hline$S$ & .018 & 36 & 1.2 & $\mathrm{U}$ & 1 & 4 & 0.00 & & $\mathrm{Y}$ & 1 & 0.87 & & 2.61 & 0.16 & 4.79 & 0. & 4.32 & 35 & .27 & 121 & 12 & 1.3 & 0 & 49 \\
\hline AGAI & 1.021 & 566 & 1.71 & $\mathrm{P}$ & 5.32 & 4 & 00 & & $\mathrm{Y}$ & 1 & 0.65 & & -5.64 & 0.10 & 4.54 & 0.2 & 3.23 & 0.18 & 13 & 52 & 18 & 1.2 & 0 & 61 \\
\hline AGAL351.026-00.321_S & 1.026 & 0.321 & 1.02 & A & -17.64 & 4 & 0.00 & 0.00 & $\mathrm{Y}$ & 1 & 0.20 & & -18.07 & 0.31 & 4.38 & 0 & 0.94 & 0.15 & 12 & 306 & 6 & 1.0 & 0 & 25 \\
\hline 51.041-00.336_S & 1.041 & 0.336 & 0.47 & A & -18.34 & 8 & 0.00 & 0.00 & $\mathrm{Y}$ & 1 & 1.65 & & -18.72 & 0.05 & 4.20 & & 8.10 & 0.22 & 16 & 0.000 & 37 & 8 & 1 & 106 \\
\hline 9_S & 1.041 & 0.579 & 5.18 & $\mathrm{P}$ & -5 & 4 & 0.00 & 0.00 & $\mathrm{Y}$ & 1 & 0.79 & 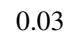 & -5.75 & .08 & 3.93 & & 3.39 & .17 & 13 & 0.002 & 0 & 1 & 1 & 32 \\
\hline $.131+00.771 \_S$ & 1.131 & & & Q & & 6 & 0.00 & 0.00 & $\mathrm{Y}$ & 1 & 1.11 & & & 08 & 4.63 & & 5.53 & .23 & 18 & & & & 0 & 57 \\
\hline 6_S & 1.139 & & & $\mathrm{Q}$ & & 8 & 0.69 & 0.28 & $\mathrm{Y}$ & 1 & 1.27 & & & 0.07 & 4.55 & & 6.66 & 27 & 18 & & 4 & 0 & 0 & 52 \\
\hline 1+00.776_S & 1.141 & & & $\mathrm{U}$ & & 6 & 0.00 & & $\mathrm{Y}$ & 1 & 0.92 & & & 0.09 & 4.18 & & & 23 & 17 & & 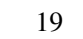 & 4 & 0 & 36 \\
\hline & .151 & & & $\mathrm{Q}$ & & 7 & 0.46 & & $y$ & 1 & & & & & & & & & & & 0 & .1 & 0 & 43 \\
\hline $\mathrm{S}$ & 9 & & & $\mathrm{U}$ & & 6 & 0.93 & & $\mathrm{Y}$ & 1 & 1.04 & & & 10 & 5.68 & 0 & 6.58 & 27 & 18 & & 4 & 9 & 1 & 55 \\
\hline & 1 & & & $F$ & & 9 & 0.27 & & Y & 1 & & & & 02 & 5.77 & 0 & 23.86 & 26 & 13 & & 1 & 8 & 1 & 175 \\
\hline _S & 3 & & 8 & L & & 9 & 0.22 & & Y & 1 & & & & 04 & 5.95 & 0.09 & 57 & 20 & 12 & & 8 & & 1 & 72 \\
\hline SS & 3 & & & $\mathrm{c}$ & & 7 & 0 & & $Y$ & 2 & 7 & & & 1 & 4.11 & & 10 & 26 & 12 & & 1 & 9 & 1 & 98 \\
\hline & 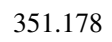 & & & $\mathrm{F}$ & & 4 & 0 & & & 1 & 9 & & & & 6.71 & & & & & & & & 1 & 69 \\
\hline S & 18 & 0 & 0 & c & -4 & 7 & 0.00 & 0. & $\mathbf{Y}$ & 1 & 2.82 & 0 & -4.61 & 0.02 & 3.86 & 0. & 12.33 & 0.17 & 12 & 0.000 & 7 & 3 & 1 & 111 \\
\hline - $S$ & 210 & 0 & & $\mathrm{~L}$ & -4.0 & 9 & 004 & 0.2 & -1 & 1 & 364 & 0 & -4.17 & 0.01 & 3.08 & 0. & 12.71 & 0.22 & .14 & 0.000 & 58 & 2.1 & 1 & 93 \\
\hline I $\mathrm{S}$ & 1.228 & 0.691 & ) & A & -4.22 & 9 & 0.39 & 0.1 & Y & 1 & 3.36 & 0 & -4.21 & 0.02 & 3.58 & 0. & 13.75 & 0.19 & 12 & 0.000 & 71 & 2 & 1 & 122 \\
\hline S & 236 & & 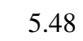 & C & -1 & 9 & 000 & 0. & 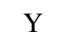 & 1 & 4.35 & 0 & -1.85 & 0.01 & 3.70 & 0. & 18.07 & 0.22 & 4 & 0.000 & 83 & 3 & 1 & 83 \\
\hline AGA & 1.244 & 9 & 4.38 & $c$ & -2.83 & 11 & 0.04 & 0.2 & $Y$ & 2 & 5.67 & 0. & -3.06 & 0.01 & 5.48 & 0. & 30.75 & 0.31 & 15 & 0.000 & 100 & 0 & 1 & 101 \\
\hline AGAL3 & 251 & 52 & 0.46 & A & -0.42 & 10 & 0.43 & 0.1 & $\mathrm{Y}$ & 1 & 4.12 & 0. & -0.78 & 0.02 & 4.48 & 0. & 20.59 & 0.20 & 13 & 0.000 & 103 & 3.7 & 1 & 105 \\
\hline AGAL3 & 2 & 64 & 44 & 0 & -2.03 & 9 & 023 & 0. & Y & 2 & 2.69 & & -2.38 & 0.02 & 4.84 & 0. & 12.61 & 0.25 & 13 & 0.002 & 51 & 4 & 1 & 30 \\
\hline AGAL3 & 30 & & & $\mathrm{U}$ & 375 & 4 & 0 & 0. & 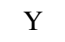 & 1 & 1.10 & 0 & -3.81 & 0.06 & 4.46 & 0 & 5.34 & 0.18 & 13 & 0.217 & 29 & 1.4 & 0 & 51 \\
\hline$\triangle \mathrm{AAI}_{3}$ & 353 & & & $\mathrm{U}$ & -1.61 & 5 & 17 & 0. & 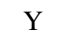 & 1 & 1.64 & 0 & -1.79 & 0.08 & 3.91 & 0. & 8.86 & 0.47 & .29 & .000 & 19 & 2.0 & 1 & 72 \\
\hline S & 1.359 & & 1.12 & $\mathrm{P}$ & -1.97 & 6 & 0.00 & 0.1 & $\mathrm{Y}$ & 1 & 1.62 & 0. & -2.06 & 0.04 & 4.62 & 0. & 8.15 & 0.20 & 14 & 00 & 40 & 3.4 & 1 & 101 \\
\hline $\mathrm{AG}$ & 2 & & 0.8 & $\mathrm{P}$ & -2.88 & 9 & 0.99 & 0. & $\mathrm{Y}$ & 1 & 3.66 & & -2.88 & 0.03 & 5.06 & 0.06 & 20.12 & 0.28 & 18 & 0.000 & 73 & 3.7 & 1 & 76 \\
\hline AGAL351.378+00.706_S & 351.378 & 0.706 & 4.58 & $\mathrm{P}$ & -4.53 & 6 & 0.13 & 0.37 & $\mathrm{Y}$ & 1 & 2.48 & 0.03 & -4.31 & 0.03 & 4.75 & 0.07 & 12.62 & 0.21 & 0.14 & 0.000 & 61 & 5.0 & 1 & 141 \\
\hline
\end{tabular}


and Figure 5 a PDR. Moreover, in Figures 6 and 7, we also show an example of when two separate components were detected along the line of sight towards an ATLASGAL clump ('A' and 'B' components), and in Figure 8, we show an example of a clump that is located in the Galaxy's Central Molecular Zone (CMZ).

In all cases, these figures show an ATLASGAL $870 \mu \mathrm{m}$ dust continuum image, two Spitzer three colour images (combining the 3-8 $\mu \mathrm{m}$ and 3-24 $\mu \mathrm{m}$ data from the GLIMPSE and MIPSGAL surveys; Benjamin et al. 2003; Carey et al. 2009), and panels showing the MALT90 spectra, fits, and residuals for each emission line. If emission was detected, the derived parameters from the best-fitting Gaussian profile are labelled, the red line shows this best-fitting profile. The $V_{\mathrm{C}}$ is marked as the solid vertical line.

Quiescent clumps (e.g., Figure 1) are categorised as bright in the $870 \mu \mathrm{m}$ dust continuum emission (top left image) but dark at 3-24 $\mu \mathrm{m}$ (top middle and right images), indicating that the dust is cold and dense. As expected, these clumps are typically detected only from the four dense gas tracers of $\mathrm{HCO}^{+}, \mathrm{HNC}, \mathrm{N}_{2} \mathrm{H}^{+}$, and $\mathrm{HCN}$ (this example also shows a marginal detection of $\mathrm{H}^{13} \mathrm{CO}^{+}$) but not detected in the high excitation lines. In this example, both the $\mathrm{N}_{2} \mathrm{H}^{+}$, and $\mathrm{HCN}$ hyperfine components were reliably fit with a three-component Gaussian profile. For all spectra towards this clump, the fit residuals are low $\left(R_{\mathrm{f}}<1.5\right)$ and the measured $V_{\mathrm{LSR}}$ are consistent with the derived $V_{\mathrm{C}}$. Moreover, consistent with its lack of star formation, the line emission from this clump has relatively narrow line-widths $\left(\sim 2.1 \mathrm{~km} \mathrm{~s}^{-1}\right)$.

In contrast, towards typical protostellar clumps (e.g., Figure 2) which show obvious IR signatures of active star formation such as extended $4.5 \mu \mathrm{m}$ emission tracing shocked gas (colour-coded as green in the top middle image) and bright $24 \mu \mathrm{m}$ emission tracing an embedded protostar (colour-coded as red in the top right image), the suite of $90 \mathrm{GHz}$ transitions are well detected, and in many cases, the emission is bright, with complex line profiles. In this example, the $\mathrm{N}_{2} \mathrm{H}^{+}$emission is well-fit by a three-component Gaussian profile. For $\mathrm{HCO}^{+}, \mathrm{HNC}$, and $\mathrm{HCN}$, however, the profiles are clearly non-Gaussian, so while a single component was found to be the 'best fit' to these profiles, there are clearly significant residuals remaining in the spectrum: in these cases, the reported $R_{\mathrm{f}}$ values are 3.8-6.0. A comparison between the profiles of the optically thick and thin transitions (i.e., $\mathrm{HCO}^{+}$and $\mathrm{H}^{13} \mathrm{CO}^{+}$or $\mathrm{HNC}$ and $\mathrm{HN}^{13} \mathrm{C}$ ) indicate that the optically thick transitions show an asymmetric selfabsorption profile and, thus, may be tracing infall/outflows motions within the gas. Moreover, the detection of the high excitation lines towards this clump (i.e., $\mathrm{HNCO}, \mathrm{CH}_{3} \mathrm{CN}$, and $\mathrm{HC}_{3} \mathrm{~N}$ ) suggests that the gas is heated by a newly formed protostar and confirms active star formation within this clump. Moreover, the detection of broad SiO emission independently confirms shocked gas, most likely due to an outflow.

Towards typical H II regions (e.g., Figure 3) which have bright 3-24 $\mu \mathrm{m}$ emission, indicative of embedded, active star formation, the $90 \mathrm{GHz}$ transitions tend to be bright, with complex line profiles, similar to protostellar clumps. Differences can be seen, however, in the relative intensities of several of the lines for these two categories (e.g., compare the line strengths of the isotopologues, higher excitation lines, and the $\mathrm{SiO}$ emission in Figures 2 and 3). These reflect different chemical and physical conditions within clumps in these two stages.

Although it is only rarely detected, $\mathrm{H} 41 \alpha$ emission was detected towards 20 clumps ( $0.6 \%$ of the full sample). Since $\mathrm{H} 41 \alpha$ traces ionised gas, presumably from an embedded high-mass star that is emitting UV radiation, its detection indicates a high-mass star with large ionising flux. These will only occur in the later phase in the evolution for these clumps. Figure 4 shows an example of a clump, and its associated IR emission, that has bright, reliable detection of $\mathrm{H} 41 \alpha\left(\mathrm{II}_{\mathrm{SNR}}=\right.$ 25).

Clumps that appear to be associated with a PDR (e.g., Figure 5) were separated into their own IR-based category, since they are likely associated with very different conditions within the gas compared to the other quiescent clumps, protostellar clumps, and $\mathrm{H}$ II regions. Because PDRs are regions illuminated along the edge of a molecular cloud by nearby UV radiation from a high-mass star, clumps located within them are likely to be externally heated and potentially dominated by shock and PDR chemistry. Indeed, in the example shown in Figure 5, although there appears to be no IR emission directly associated with the bright dust emission, we see line emission from many of the higher excitation transitions and $\mathrm{SiO}$ which indicates that the gas is heated and shocked. While these clumps are valuable to study in detail to learn about star formation that is potentially triggered on the edge of a molecular cloud, we exclude these clumps from the analysis and discussion of evolution, since it is unclear if they indicate a distinct phase in the evolutionary sequence for these clumps.

Figures 6 and 7 show an example of a clump (in this case, a protostellar clump) that has two distinct velocity components detected towards it. Component A, shown in Figure 6, is well detected in the lines of $\mathrm{HCO}^{+}, \mathrm{HNC}, \mathrm{N}_{2} \mathrm{H}^{+}$, and $\mathrm{HCN}$ (and $\mathrm{C}_{2} \mathrm{H}$ ), while component $\mathrm{B}$, shown in Figures 7 , is well detected in the first three of these lines. In all cases, the profiles are well-fit and the two components well-separated in velocity.

The final example, in Figure 8, shows the line emission towards a clump located in the Galaxy's CMZ. Note the very broad line profiles, $\Delta V \sim 17-27 \mathrm{~km} \mathrm{~s}^{-1}$, which are due to the high levels of turbulence that characterise clumps located in the CMZ. While this clump appears to be IR-dark and, as such, is classified as 'quiescent', many of the higher excitation lines, including $\mathrm{SiO}$, are also detected towards it. Since the bulk of the gas in the CMZ has temperatures of $\sim 80 \mathrm{~K}$ and densities $>10^{4} \mathrm{~cm}^{-3}$ (Walmsley et al. 1986; Ao et al. 2013), many of the higher excitation lines (like those covered by MALT90) show bright, widespread emission across the region. This has led to the speculation that 
perhaps shock chemistry dominates in the CMZ (Wilson et al. 1982; Martín-Pintado et al. 2001). As such, we also exclude all clumps that fall within $\pm 10^{\circ}$ in Galactic longitude of the Galactic centre from the analysis and discussion of evolution since their chemistry is likely very different from the rest of the clumps located throughout the Galactic disk.

\section{ENSEMBLE PROPERTIES}

\subsection{The MALT90 sample: representative of typical cluster-forming clumps}

ATLASGAL was a systematic, unbiased survey of dust continuum emission with the goal of identifying and characterising high-mass clumps within the Galaxy (Schuller et al. 2009). As such, the catalogues derived from this survey represent an excellent database for detailed studies of these clumps.

The MALT90 survey mapped 3246 clumps that were identified within ATLASGAL (within the Galactic longitude range of -60 to $+20^{\circ}$ ). To demonstrate that the MALT90selected clumps are representative of the larger ATLASGAL sample, Figure 13 shows histograms of the $870 \mu \mathrm{m}$ peak flux from ATLASGAL for the clumps included within MALT90 (thin histogram), along with the ATLASGAL sample for comparison (thick histogram). MALT90 observed almost all $(\sim 90 \%)$ of the ATLASGAL clumps with fluxes $>1 \mathrm{Jy}$, and $\sim 60 \%$ of the ATLASGAL clumps with fluxes between 0.25 and $1 \mathrm{Jy}$. Those ATLASGAL clumps that were not included within MALT90 are typically located within the Galaxy's CMZ or in the first Galactic quadrant: MALT90's primary goal was to cover clumps within the fourth Galactic quadrant.

The panels of Figure 13 also shows the $870 \mu \mathrm{m}$ flux distribution for the MALT90 clumps separated by the number of lines detected towards them (i.e., NL $\geq 1,4,6$, and 8 , from upper to lower panels, respectively; dotted histograms). The clumps with no detected lines (160 in total) are typically the faintest $870 \mu \mathrm{m}$ clumps (see the upper panel of Figure 13), while those with multiple lines detected towards them are amongst the brightest $870 \mu \mathrm{m}$ clumps (see the lower panels in Figure 13).

The trend of brighter $870 \mu \mathrm{m}$ clumps having a higher number of lines detected towards them is also seen when the MALT90 sample is separated into the IR-based categories (see Figure 14). Regardless of the category, the brightest $870 \mu \mathrm{m}$ clumps within each group have a higher fraction of lines detected towards them. Moreover, those that have no detected emission tend to be at the fainter end of the ATLASGAL flux distribution.

\subsection{Detection statistics}

In addition to listing information about the emission lines observed as part of the MALT90 survey, Table 1 also sum-

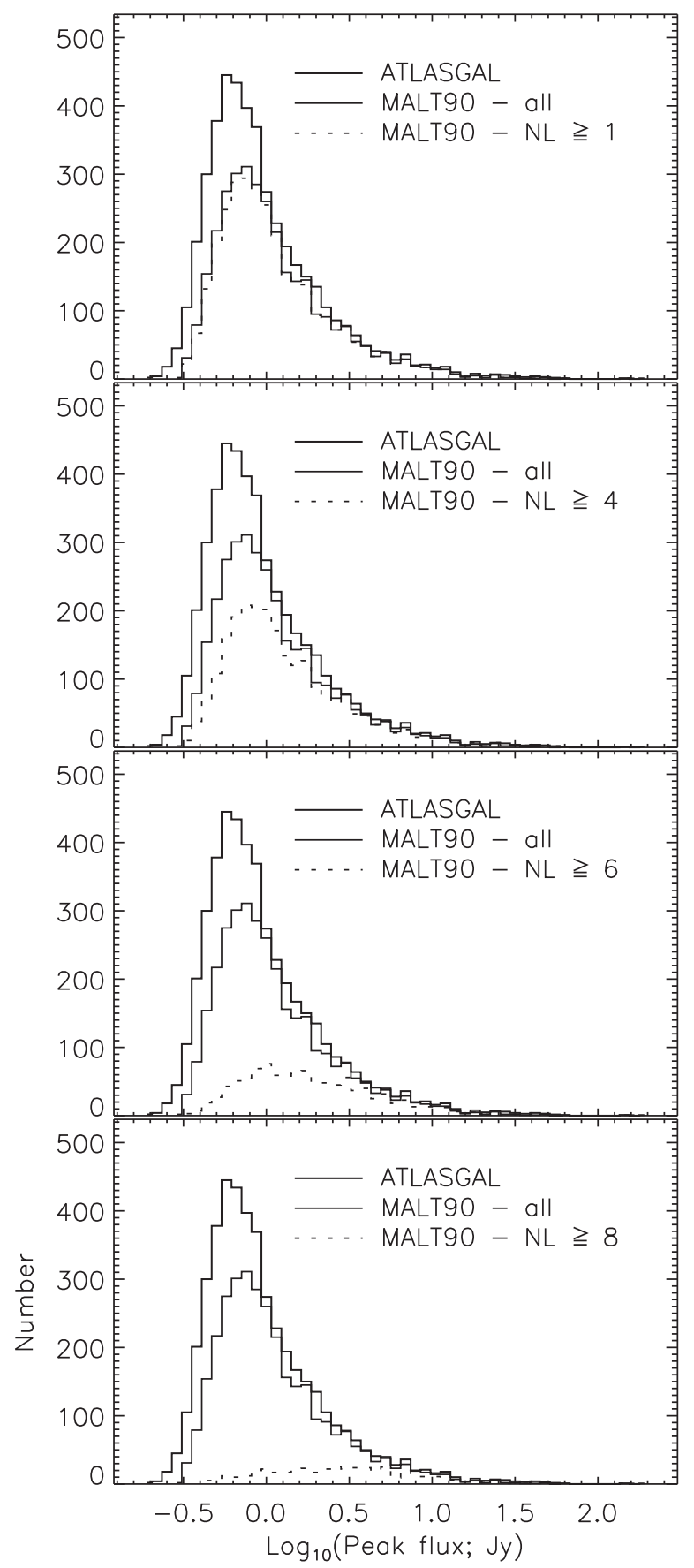

Figure 13. Histograms of the $870 \mu \mathrm{m}$ peak flux for clumps covered by the MALT90 survey (thin histogram). In all panels, clumps from the ATLASGAL survey are shown as thick histograms, while the dotted histograms show the flux distribution of those clumps covered by MALT90 survey and detected in more than the labelled number of lines (i.e. $\mathrm{NL} \geq 1,4,6$, and 8 ; upper to lower panels, respectively).

marises the detection rates for each line. ${ }^{3}$ We find that the detection rates are highest for the four (1-0) molecular transitions of $\mathrm{HCO}^{+}, \mathrm{HNC}, \mathrm{N}_{2} \mathrm{H}^{+}$, and $\mathrm{HCN} \quad(\sim 77-89 \%)$. A

\footnotetext{
${ }^{3}$ These percentages are calculated with respect to the 3246 ATLASGAL clumps covered by MALT 90 .
} 

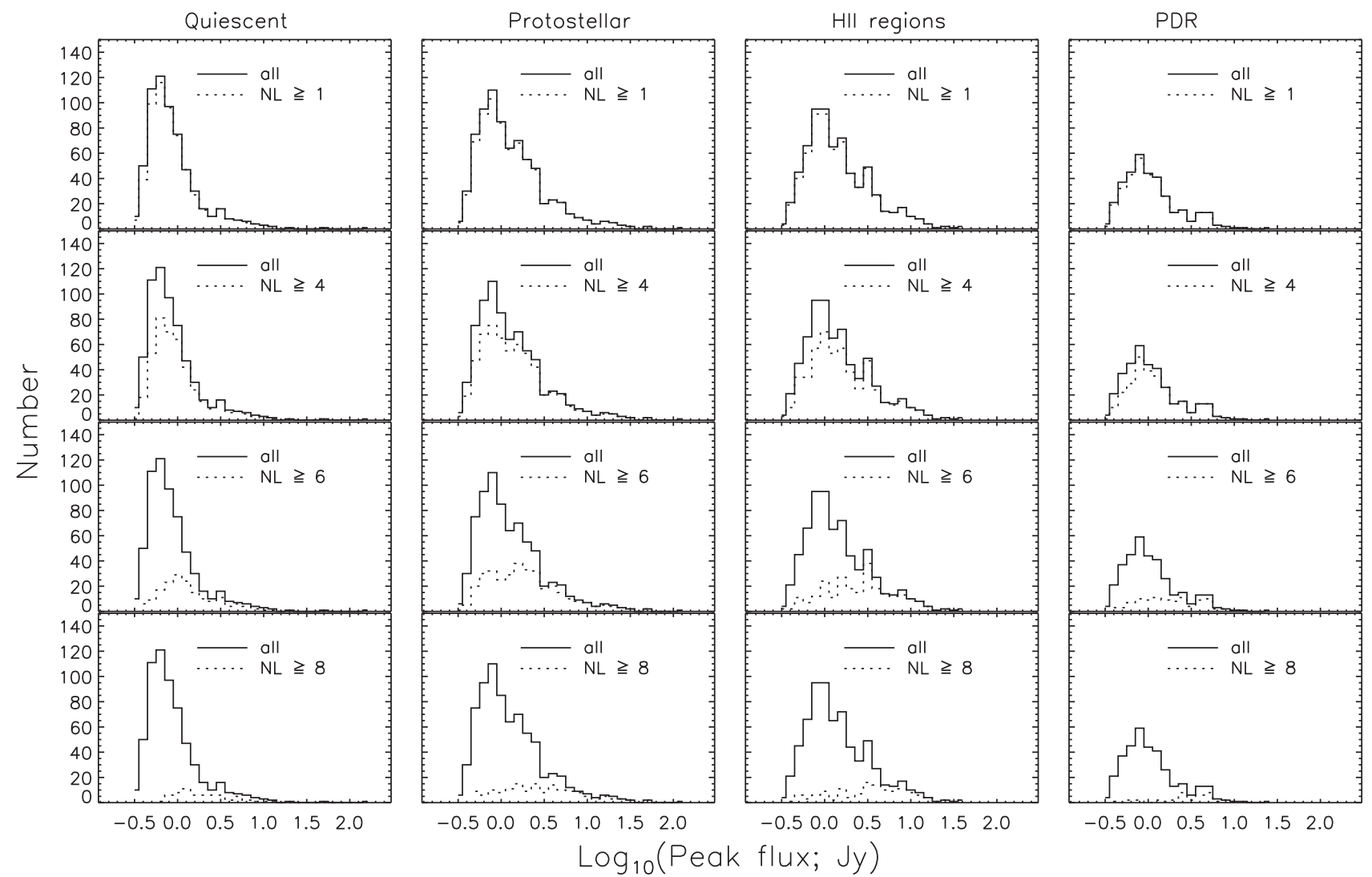

Figure 14. Histograms of the $870 \mu \mathrm{m}$ peak flux for clumps covered by the MALT90 survey, separated by the IR-based categories (quiescent, protostellar, $\mathrm{H}$ II regions, and PDRs, left to right, respectively). In all panels, clumps included within MALT90 are shown as thin histograms, while the dotted histograms show the flux distribution of those clumps covered by MALT90 survey and detected in more than the labelled number of lines (i.e. NL $\geq 1,4,6$, and 8; upper to lower panels, respectively).

small number of clumps are detected in the lines of ${ }^{13} \mathrm{C}^{34} \mathrm{~S}$, $\mathrm{HC}^{13} \mathrm{CCN}$, and $\mathrm{H} 41 \alpha(8,8$, and 20 respectively); the number of clumps detected in each line is listed in Table 1.

Figure 15 shows that most clumps $(\sim 95 \%)$ are detected in at least one line. Just over half of the clumps $(\sim 53 \%)$ are detected in four or more transitions, while only one clump is detected in 13 transitions (AGAL351.444+00.659_S).

Figure 16 shows the normalised cumulative distribution of the $T_{\mathrm{SNR}}$ and $\mathrm{II}_{\mathrm{SNR}}$ for each of the emission lines. The vertical dotted lines in all panels mark the cuts used to define detections for inclusion in the catalogue (i.e., $T_{\mathrm{SNR}}>1$ and $\mathrm{II}_{\mathrm{SNR}}>3$, in the case of marginal detections, or $T_{\mathrm{SNR}}>1$ and $\mathrm{II}_{\mathrm{SNR}}>4$ in the case of reliable detections). Flat curves in these plots (e.g., the $\mathrm{II}_{\mathrm{SNR}}$ curves for $\mathrm{HCO}^{+}, \mathrm{HNC}, \mathrm{N}_{2} \mathrm{H}^{+}$, $\mathrm{HCN}, \mathrm{SiO}, \mathrm{HNCO}, \mathrm{CH}_{3} \mathrm{CN}$, and $\mathrm{H} 41 \alpha$ ) imply that the inclusion or exclusion of detections is insensitive to the exact choice of the selection criteria. In contrast, for curves that rise steeply (e.g., the $\mathrm{II}_{\mathrm{SNR}}$ curves for $\mathrm{C}_{2} \mathrm{H}, \mathrm{H}^{13} \mathrm{CO}^{+}, \mathrm{HC}_{3} \mathrm{~N}$, $\mathrm{HN}^{13} \mathrm{C}$, and ${ }^{13} \mathrm{CS}$ ), the exact choice of the selection criteria does significantly affect the number of detections that are included in the catalogue. To avoid unnecessary exclusion of possible detections, we mark in the catalogue all detections with a derived $3<\mathrm{II}_{\mathrm{SNR}}<4$ as 'marginal'. Note that the

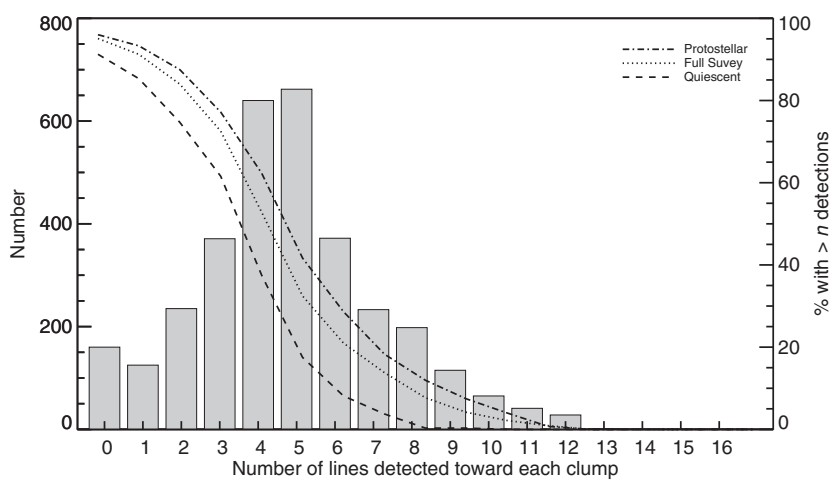

Figure 15. Number of clumps plotted as a function of the number of emission lines detected towards each (bar plot; left axis). The dotted line (right axis) shows the percentage of clumps with $>n$ detections in the full survey. Most clumps $(\sim 95 \%)$ are detected in one or more of the dense gas tracers, $\sim 53 \%$ of the clumps have detections from four or more emission lines, while one clump is detected in 13 lines. Also, overlaid are the percentages of quiescent and protostellar clumps with $>n$ detections (right axis; dashed and dot-dashed lines, respectively). Clumps with active star formation (indicated by the 'protostellar' curve) have a both a higher percentage of detections and more lines detected towards them compared to the full survey and those clumps that appear to be more quiescent. 

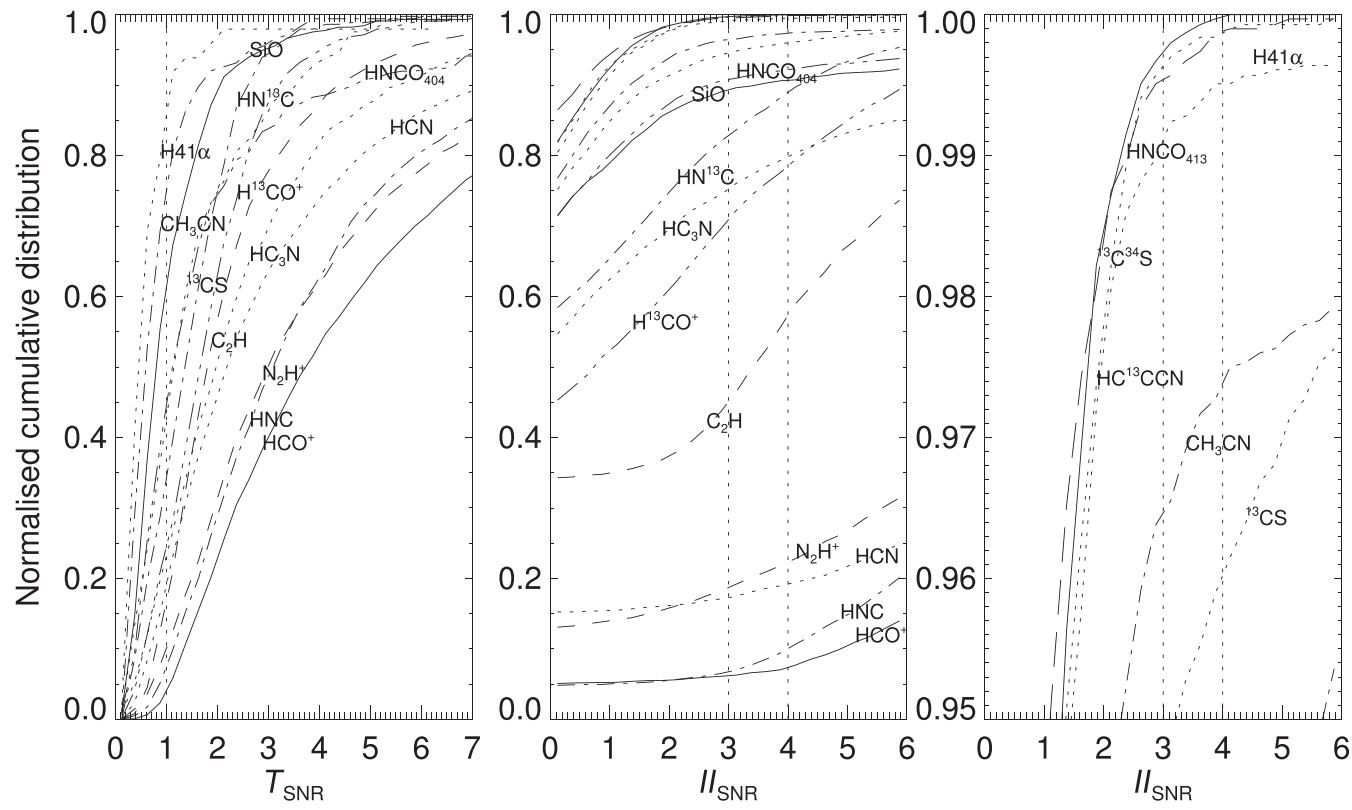

Figure 16. Normalised cumulative distributions of $T_{\mathrm{SNR}}$ (left panel) and $\mathrm{II}_{\mathrm{SNR}}$ (middle and right panels). In all panels, curves are labelled with the name of corresponding emission line. The vertical dotted lines mark the selection criteria imposed for detections to be included within the catalogue: $T_{\mathrm{SNR}}>1$ and an $\mathrm{II}_{\mathrm{SNR}}>3$ for 'marginal' or $>4$ for 'reliable' detections, respectively. Flat curves imply that the inclusion/exclusion of detections is insensitive to the exact choice of the selection criteria (i.e., in the $\mathrm{II}_{\mathrm{SNR}}$ plot for $\mathrm{HCO}^{+}, \mathrm{HNC}, \mathrm{N}_{2} \mathrm{H}^{+}, \mathrm{HCN}, \mathrm{SiO}, \mathrm{HNCO}, \mathrm{CH}_{3} \mathrm{CN}$, and $\mathrm{H} 41 \alpha$ ). In contrast, for curves that rise steeply, the exact choice of the $\mathrm{II}_{\mathrm{SNR}}$ ( 3 or 4 ) does significantly affect the number of reported detections included in the catalogue (i.e., for $\mathrm{C}_{2} \mathrm{H}, \mathrm{H}^{13} \mathrm{CO}^{+}, \mathrm{HC}_{3} \mathrm{~N}, \mathrm{HN}^{13} \mathrm{C}$, and ${ }^{13} \mathrm{CS}$ ).

selection criteria of $T_{\mathrm{SNR}}>1$ has little effect, since almost all lines have values greater than this. However, this cut was imposed as part of the selection of reliable detections to avoid the inclusion of very broad lines that have a high II, but are not discernible above the noise in $T_{\mathrm{A}}^{*}$ (i.e., any remaining slowly varying baselines that were inadequately removed).

The upper panel of Figure 17 shows the percentage of clumps that were detected in each of the emission lines. As expected, the highest detection rates of the lines covered by MALT90 arise from the (1-0) transitions of $\mathrm{HCO}^{+}$, $\mathrm{HNC}$, $\mathrm{N}_{2} \mathrm{H}^{+}$, and $\mathrm{HCN}$. Of the isotopologues, $\mathrm{H}^{13} \mathrm{CO}^{+}$has the highest detection rate, followed by $\mathrm{HN}^{13} \mathrm{C}$. The molecule $\mathrm{C}_{2} \mathrm{H}$ is also frequently detected towards the clumps $(\sim 51 \%)$.

The middle panel of Figure 17 shows the percentage of clumps with detections for each emission line, separated out by the number of Gaussian profiles that were fit to the spectrum (i.e., 1, 2, or 3; dark grey, grey, and light grey bars, respectively). In the majority of cases for transitions without hyperfine splitting, the best fit was found when fitting a single Gaussian profile to the emission (dark grey bars). For transitions with hyperfine splitting $\left(\mathrm{N}_{2} \mathrm{H}^{+}, \mathrm{HCN}\right.$, and $\mathrm{C}_{2} \mathrm{H}$ ), the best fit was most often found when it included the known hyperfine structure (light grey bars). However, in a small number of cases, a single Gaussian profile was found to produce the best fit; this occurred when individual hyperfine components could not be easily separated, either due to broad line-widths or low signal to noise $(\sim 7 \%, \sim 15 \%$ and $\sim 2 \%$ for $\mathrm{N}_{2} \mathrm{H}^{+}, \mathrm{HCN}$, and $\mathrm{C}_{2} \mathrm{H}$, respectively).
The lower panel of Figure 17 shows the percentage of clumps with detections for each molecular transition, separated by the significance of the residual that remains after the best-fitting profile(s) are removed. Dark grey bars show the percentage of clumps for which the residuals are low (i.e., $\mathrm{HR}=0$ ); light grey bars indicate the percentage where the residuals are high $(\mathrm{HR}=1)$. The spectra from $\mathrm{HCO}^{+}$show the highest percentage of those with HR, which is expected since this molecular transition typically has the highest optical depth of those included in the survey (e.g., Sanhueza et al. 2012; Hoq et al. 2013). As such, $\mathrm{HCO}^{+}$is therefore an excellent tracer of outflow and infall motions that give rise to non-Gaussian line profiles.

\subsection{Distributions of the derived parameters}

To determine whether the various molecular transitions are tracing similar or distinct physical regions, we have plotted in Figure 18 the distribution functions of the ratio of II to the mean II (II/mean(II); left), line-width ( $\Delta V$; middle), and the difference between the derived velocity and the $V_{\mathrm{C}}\left(\mathrm{V}-V_{\mathrm{C}}\right.$; right), for a subset of the detected molecular transitions.

As might be expected for optically thicker molecular transitions (e.g., $\mathrm{HCO}^{+}, \mathrm{HNC}, \mathrm{N}_{2} \mathrm{H}^{+}$, and $\mathrm{HCN}$ ) show a relatively broader II distribution compared to the optically thinner molecular transitions (e.g., $\mathrm{H}^{13} \mathrm{CO}^{+}, \mathrm{HN}^{13} \mathrm{C}$, and ${ }^{13} \mathrm{CS}$ ). Since an optically thick transition will trace gas temperature, which will span a broad range, a broad distribution in their 

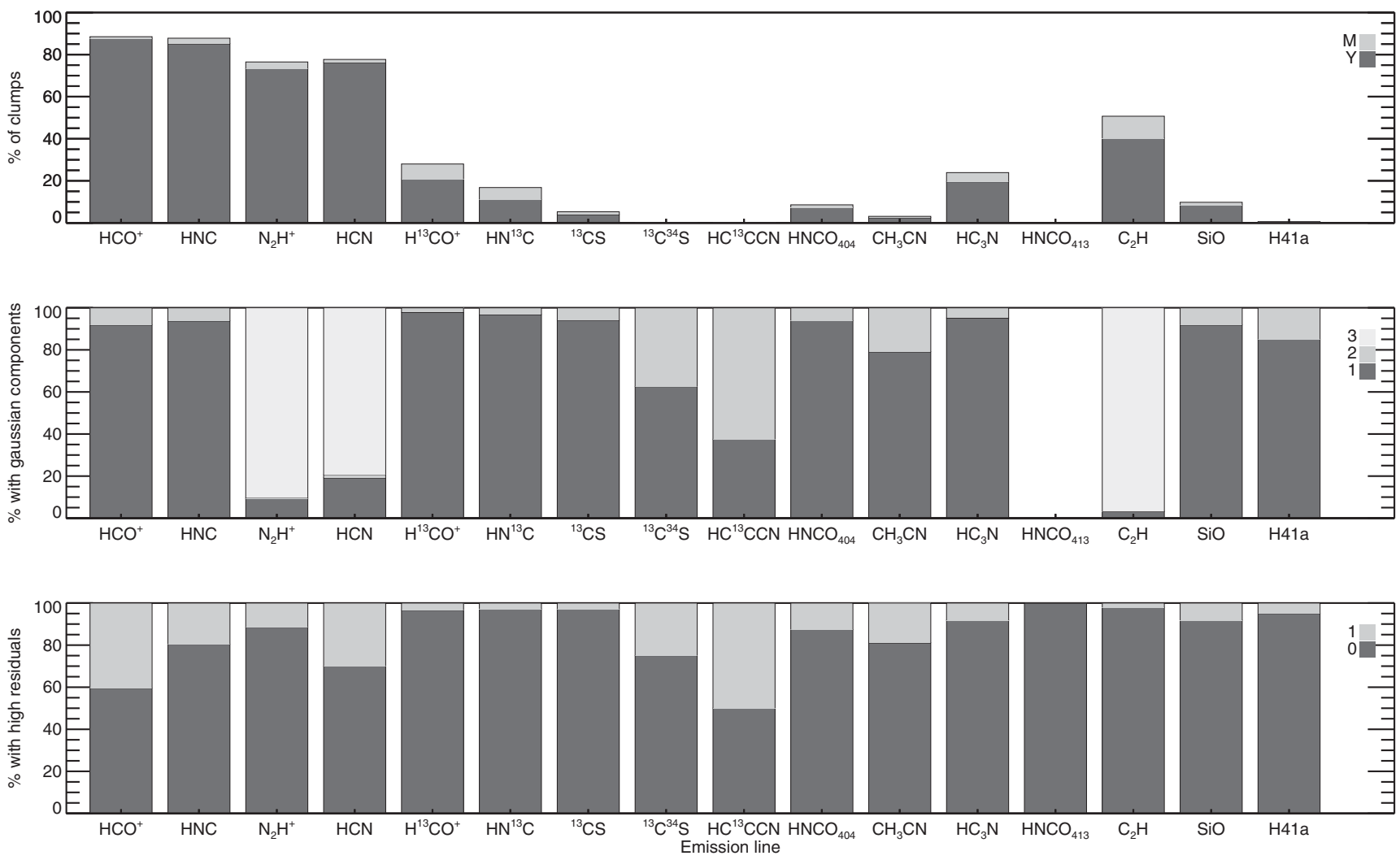

Figure 17. Detection rates (upper panel; separated by the significance of the detection, ' $Y$ ' or ' $M$ '), the number of Gaussian profiles in the best fit for these detections (middle panel; 1,2 , or 3 ), and the significance of the residuals in the fit range (lower panel; $R_{\mathrm{f}}<1.5$, HR $=0$ or $R_{\mathrm{f}}>1.5$, HR $=1$ ) for all emission lines. Note for HNCO 4(1,3)-3(1,2), since there was no emission detected towards any of the clumps, the percentages in the top two panels are zero; however, the lower panel (which plots the residual in the fit range) shows that all the spectra have low residuals, which is consistent with non-detections in all cases.

integrated intensities would be expected. Moreover, since the rarer isotopologues will be optically thin and, thus, sensitive to column density only, we would expect a narrower range in the distribution in their integrated intensities. The higher excitation lines (i.e., $\mathrm{HNCO}, \mathrm{CH}_{3} \mathrm{CN}, \mathrm{HC}_{3} \mathrm{~N}, \mathrm{C}_{2} \mathrm{H}$, and $\mathrm{SiO}$ ) all show a very broad distribution in their integrated intensities. This likely reflects the very different conditions (e.g., shocks, high excitation temperatures) of the gas within which these molecular lines are excited.

The line widths are in general narrowest for the isotopologues (e.g., $\mathrm{H}^{13} \mathrm{CO}^{+}, \mathrm{HN}^{13} \mathrm{C},{ }^{13} \mathrm{CS}$ ), compared to the optically thicker transitions (e.g., $\mathrm{HCO}^{+}, \mathrm{HNC}$, and $\mathrm{HCN}$ ). Since the optically thick transitions will be tracing material on larger scales compared with the optically thinner transitions, this trend would be expected. Moreover, opacity broadening would also preferentially broaden the line-widths of the higher opacity transitions. The largest linewidths are found for the highest excitation energy transitions, which tend to be excited in regions of active star formation (e.g., $\mathrm{CH}_{3} \mathrm{CN}$, $\mathrm{HNCO}$, and $\mathrm{SiO}$ ). In particular, $\mathrm{SiO}$ has broad line-widths since the molecule is associated with shocks. The broad line widths of $\mathrm{HNCO}$ and $\mathrm{CH}_{3} \mathrm{CN}$ suggests that these molecular transitions are also shock-tracers. The broadest line-widths, i.e. $>10 \mathrm{~km} \mathrm{~s}^{-1}$, arise from the subset of clumps located within the Galaxy's CMZ where high levels of turbulence and shocks dominate.

Finally, the small difference between the derived velocity and the $V_{\mathrm{C}}$ within individual clumps reveals that the molecular emission is generally well centred at the $V_{\mathrm{C}}$. This implies that the emission from the various molecules arise from the same physical regions. The one exception is $\mathrm{CH}_{3} \mathrm{CN}$, for which the distribution is centred at a velocity about $-4 \mathrm{~km} \mathrm{~s}^{-1}$ from the derived $V_{\mathrm{C}}{ }^{4}$ The broadest distribution in the derived velocities relative to the $V_{\mathrm{C}}$ also occurs for the transitions with the highest line-widths: $\mathrm{CH}_{3} \mathrm{CN}, \mathrm{HNCO}$, and $\mathrm{SiO}$. This may indicate distinctly different velocities of the shocked gas.

\subsection{Trends as a function of evolution}

We use the IR-based categories to broadly represent three stages in the evolution of a clump with increasing levels

\footnotetext{
${ }^{4}$ For this molecular transition, we used the rest frequency of the unspilt 5(1)-4(1) line $(91.985313 \mathrm{GHz})$, rather than the brightest hyperfine component (the $F=5-5$ transition, at $91.98403 \mathrm{GHz}$ ). Since the emission will be dominated by the brightest hyperfine component, the resulting fitting parameters will be offset in velocity: the difference should be $\sim 4 \mathrm{~km} \mathrm{~s}^{-1}$.
} 

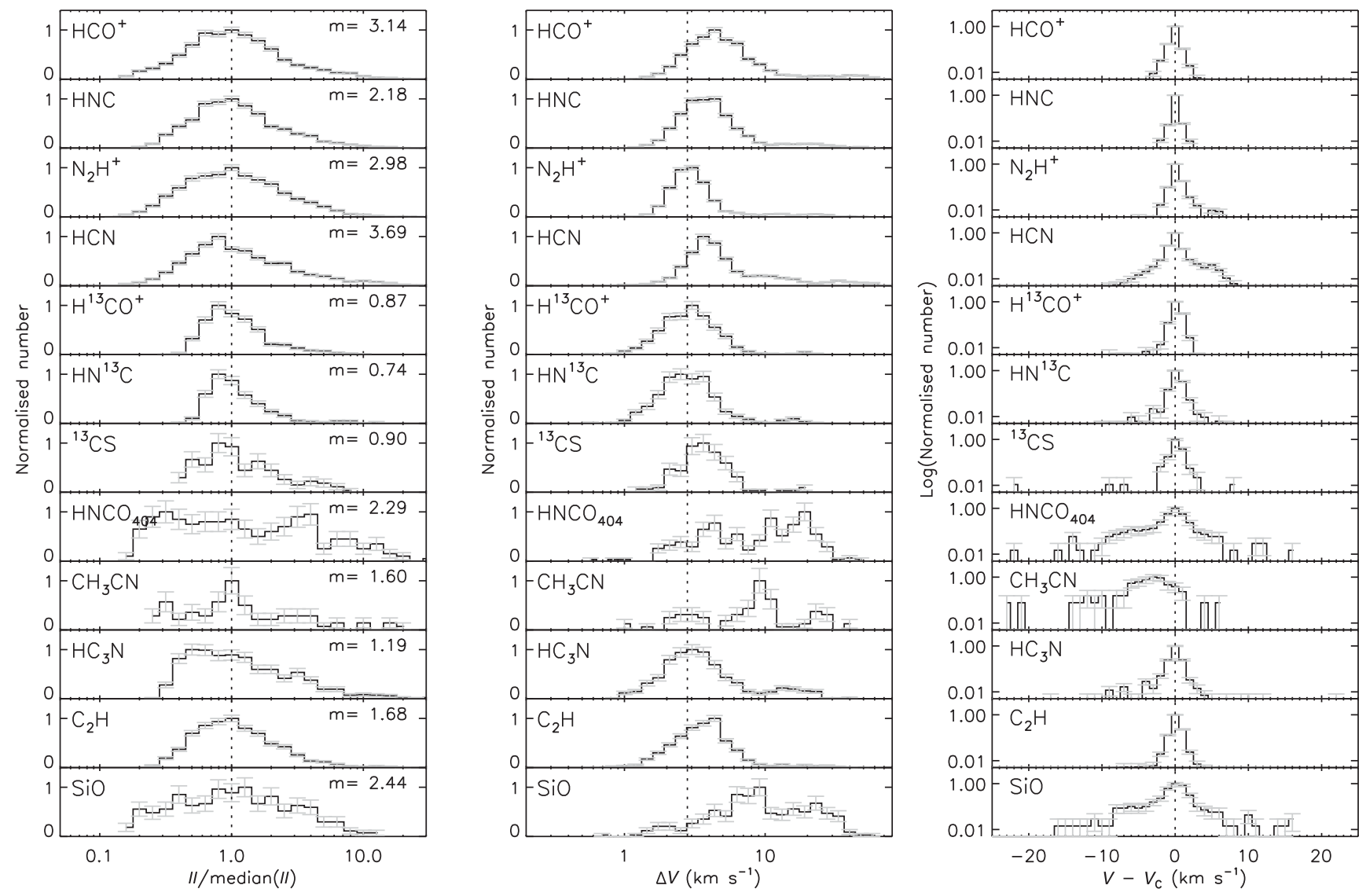

Figure 18. Histograms of the integrated intensity (II, relative to the median, left), line width ( $\Delta V$, middle), and $V-V_{\mathrm{C}}$ (right) for most of the emission lines (we only include in these plots those emission lines with more than 50 detections within the survey, see Table 1). The vertical dotted lines mark a value of 1.0 for the ratio of II to median(II), a line-width of $2.8 \mathrm{~km} \mathrm{~s}^{-1}$, (the mean value derived from $\mathrm{N}_{2} \mathrm{H}^{+}$), and a value of 0 for the velocity difference $\left(\mathrm{V}-V_{\mathrm{C}}\right.$ ), respectively. They are included for ease of comparison between the panels. Note that the $y$-axes for the right column are in a logarithmic scale. The error bars show the $\sqrt{N}$ uncertainties.

of star formation; quiescent clumps represent an early phase before star formation has begun, protostellar clumps show the initial signatures of active star formation, while $\mathrm{H}$ II regions represent a later stage when young, high-mass stars have already formed. Given the harsh environment and complexity of the chemistry within the Galaxy's CMZ compared to the Galactic disk, we exclude from the plots and analysis in this section all clumps that fall within $\pm 10^{\circ}$ in Galactic longitude of the Galactic centre.

Also overlaid on Figure 15 are the percentages of quiescent and protostellar clumps with $>n$ detections (right axis; dashed and dot-dashed lines, respectively). For simplicity, we include on this plot only curves for the quiescent and protostellar clumps: the sample of $\mathrm{H}_{\text {II }}$ regions show a similar curve to the protostellar clumps. We find that clumps with active star formation have a higher percentage of the number of lines detected compared to the full survey and those clumps that are more quiescent. Moreover, the protostellar clumps and $\mathrm{H}_{\text {II }}$ regions are detected in four or more lines more frequently ( $\sim 62$ and $\sim 58 \%$, respectively) compared to the full survey $(\sim 53 \%)$ and the quiescent clumps $(\sim 38 \%)$. While it may reflect sensitivity biases with detections of both active star formation and line emission with increasing distance, this trend with evolution is expected since the gas is likely hotter and denser in the more evolved star-forming clumps and, thus, more readily detected in the $90 \mathrm{GHz}$ lines. Indeed, a similar trend of increasing dust temperature in these clumps as a function of their evolutionary stage was confirmed in Guzmán et al. (2015).

Figure 19 shows the detection rates for most emission lines as a function of the IR-based category for each clump. The categories are shown on the $x$-axis increasing in evolutionary sequence from left to right: quiescent clumps, protostellar clumps, and $\mathrm{H}_{\mathrm{II}}$ regions. Shown as the dotted line in each panel is the percentage of clumps detected within the survey for each of the classifications $(19,22$, and $21 \%$, respectively). The detection rates within each category for the four molecular transitions of $\mathrm{HCO}^{+}, \mathrm{HNC}, \mathrm{N}_{2} \mathrm{H}^{+}$, and $\mathrm{HCN}$ follows well the overall detection rates for the full survey (see the top four panels in Figure 19). In contrast, the isotopologues and the higher excitation lines (e.g., $\mathrm{H}^{13} \mathrm{CO}^{+}$, $\mathrm{HN}^{13} \mathrm{C}, \mathrm{HNCO}, \mathrm{CH}_{3} \mathrm{CN}, \mathrm{HC}_{3} \mathrm{~N}$, and $\mathrm{SiO}$ ) are typically detected less frequently towards 'quiescent' clumps and more frequently towards those that are actively star forming (i.e., 


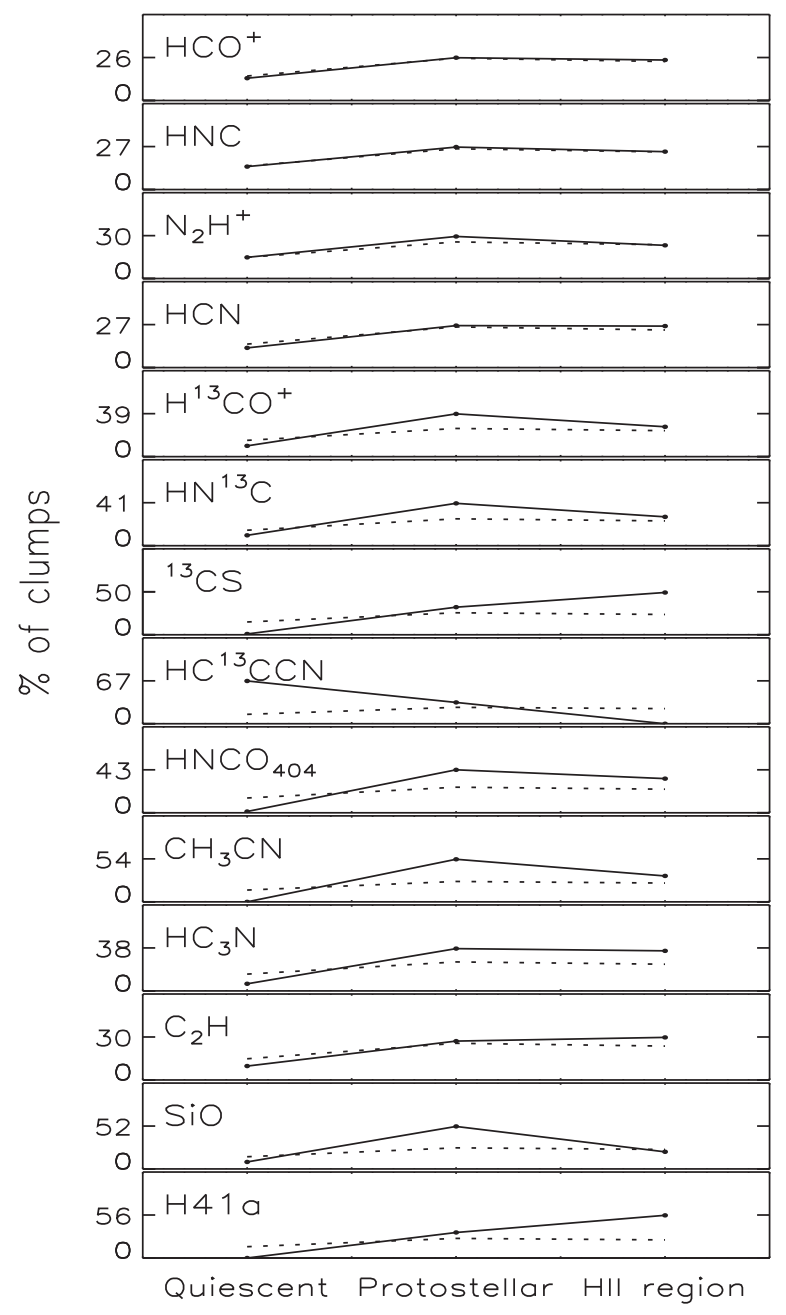

Figure 19. Detection rates for most emission lines as a function of IR-based category $\left(\mathrm{HNCO} 4(1,3)-3(1,2)\right.$ and ${ }^{13} \mathrm{C}^{34} \mathrm{~S}$ were not plotted here since they had 0 and 9 detections within the survey, respectively) for clumps located outside of the CMZ (i.e., $\pm 10^{\circ}$ of the Galactic centre). The dotted line in all panels shows the percentage of clumps detected from the survey as a whole within each of the classifications $(19,22$, and $21 \%$, respectively).

the 'protostellar' clumps and $\mathrm{H}_{\text {II }}$ regions) compared to the overall survey.

These trends are exactly what is expected if these categories reflect the evolution of the clumps: as the level of star-formation activity increases, the gas will become hotter and denser and, thus, the tracers of these conditions will be more readily detected. Indeed, this evolutionary sequence is reinforced by the clear trend in the detection rates for $\mathrm{H} 41 \alpha$ emission (see the lower panel of Figure 19). Since this emission traces ionised gas, presumably from an embedded high-mass star, it should only be observed when the central high-mass star has turned on, in the later $\mathrm{H}_{\text {II }}$ region phase.

To assess whether the ensemble of properties for the different molecular transitions varies with evolutionary stage, Figure 20 compares ratios of II (left column), ratios of linewidths (middle column), and the differences of the central velocity (right column) for various pairs of transitions as a function of the IR-derived categories for the clump. Since ratios are distance independent, they can be more reliable probes of the ensemble properties of the clumps (compared to detection statistics alone). We include in this plot a selection of pairs of transitions that both show clear trends, and for comparison, several that do not. The latter were included to demonstrate that the trends are related to particular molecules.

These plots reveal several interesting trends. First, the II ratios of $\mathrm{HCO}^{+} / \mathrm{HNC}$ and $\mathrm{HCN} / \mathrm{HNC}$ increase with evolutionary stage (left column). These trends probably indicate the fact that HNC is relatively more abundant in colder, less evolved clumps than those that show active star formation (e.g., see Hoq et al. 2013). Second, the II ratios of $\mathrm{HCO}^{+} / \mathrm{N}_{2} \mathrm{H}^{+}, \mathrm{HNC} / \mathrm{N}_{2} \mathrm{H}^{+}$, and $\mathrm{C}_{2} \mathrm{H} / \mathrm{N}_{2} \mathrm{H}^{+}$are typically higher in the $\mathrm{H}_{\mathrm{II}}$ region phase, indicating that $\mathrm{N}_{2} \mathrm{H}^{+}$is relatively brighter in the colder earlier stages, and is consistent with the notion that $\mathrm{N}_{2} \mathrm{H}^{+}$resists freezing onto dust grains more effectively than other molecules and its chemical destruction in the warm ISM (Stephens et al. 2015; Sanhueza et al. 2012).

The $\mathrm{HCO}^{+} / \mathrm{H}^{13} \mathrm{CO}^{+}$and $\mathrm{HNC} / \mathrm{HN}^{13} \mathrm{C}$ II ratios also tend to increase with increasing evolutionary stage. We surmise that this trend may indicate either less self-absorption as the clumps evolve or that the optical depth decreases as the clumps evolve. The latter is consistent with both our measurements of $\tau$ derived from the $\mathrm{N}_{2} \mathrm{H}^{+}$emission (see Figure 21) and recent results, for example, Guzmán et al. (2015), which indicate lower clump column densities in the more evolved stages.

The ratios of line-widths show no obvious trends with evolution (middle column). However, differences in the derived velocities (right column) clearly show that the $\mathrm{HCO}^{+}$emission is blueshifted with respect to the emission from the other molecular transitions (e.g., $\mathrm{HNC}, \mathrm{N}_{2} \mathrm{H}^{+}$, and $\mathrm{SiO}$ ): the difference is largest at the earliest quiescent stage and monotonically decreases as the clumps evolve. Especially, striking is the trend in the $\mathrm{HCO}^{+}-\mathrm{H}^{13} \mathrm{CO}^{+}$velocities. This result suggests that the optically thickest $\mathrm{HCO}^{+}$emission shows a 'blue-red asymmetry' that indicates overall collapse. This trend was noted from a subset of the MALT90 survey data (He et al. 2015). The blue-red asymmetry of the $\mathrm{HCO}^{+}$emission detected from the full survey will be discussed in a future paper (Jackson et al. in preparation).

\section{SUMMARY}

Targeting 3246 high-mass clumps identified in the ATLASGAL $870 \mu \mathrm{m}$ Galactic plane survey that span a range in star-formation activity, the MALT90 survey aims to characterise the physical and chemical evolution of dense, highmass clumps. By simultaneously mapping emission from 16 different species that trace specific physical and chemical conditions, the MALT90 survey provides a wealth of information about the global characteristics of the gas within 

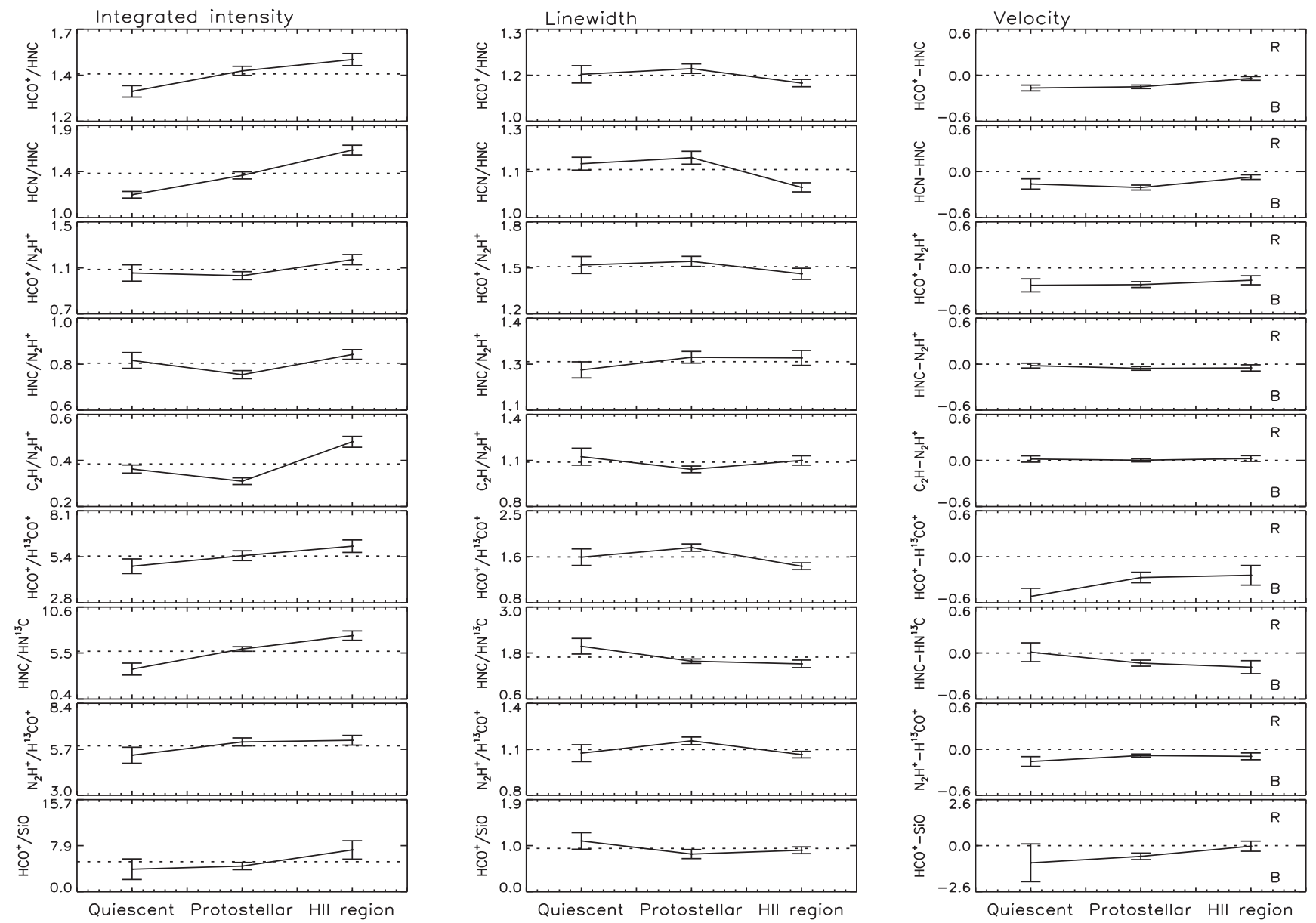

Figure 20. Trends as a function of IR-based category for clumps located outside of the CMZ (i.e., $\pm 10^{\circ}$ of the Galactic centre). Left column: median in the distribution of the ratio of integrated intensities (the dotted lines mark the mean value of the plot range and are included to help identify trends). Middle column: median in the distribution of the ratio of derived linewidths (the dotted lines mark the mean value of the plot range and are included to help identify trends). Right column: median in the distribution of the velocity differences (the dotted lines mark the zero point, the plot ranges are symmetric about zero, and labels of ' $\mathrm{R}$ ' and ' $\mathrm{B}$ ' are included to indicate the direction of any red/blue asymmetries). In all plots, the error bars show the uncertainty in the median value as calculated by bootstrap resampling with replacement (e.g., Simpson \& Mayer-Hasselwander 1986).

these clumps. This paper describes the first catalogue from MALT90 generated to summarise the properties of the emission detected towards each of the clumps.

In order to characterise the detected emission towards each clump, we have first extracted a single spectrum towards each ATLASGAL $870 \mu \mathrm{m}$ dust continuum peak for each of the emission lines, and have then fit each of these spectra with either a single or multiple Gaussian profile(s). Having determined the best-fitting profile, we report in the catalogue 16 derived values and flags for each of the 16 emission lines. We describe the catalogue entries and include a subset of its content here, the full catalogue contains 263 columns for each of the 3556 entries and is available online at www. malt90.catalog.location.

Using synthetic spectra, we estimate that the catalogue has: a completeness of $>95 \%$ for detections with a measured $T_{\mathrm{A}}^{*}>0.4 \mathrm{~K}$ and $>99 \%$ for detections with a $T_{\mathrm{A}}^{*}>0.6$ $\mathrm{K}$, a probability of a false-positive detection of $<0.3 \%$ for an $\mathrm{II}_{\mathrm{SNR}}>3$, and a relative uncertainty of the peak inten- sity, II, and linewidth of $<20 \%$ over the range of detection criteria.

The detection rates are highest for the (1-0) molecular transitions of $\mathrm{HCO}^{+}, \mathrm{HNC}, \mathrm{N}_{2} \mathrm{H}^{+}$, and $\mathrm{HCN}(\sim 77-89 \%)$. The majority of clumps $(\sim 95 \%)$ are detected in at least one of the lines, just over half of the clumps $(\sim 53 \%)$ are detected in four or more lines, while only one clump is detected in 13 lines. We find that $\mathrm{HCO}^{+}$spectra typically show nonGaussian profiles (indicated by a HR in the spectrum after the best-fitting Gaussian is subtracted), which is expected since this molecular transition typically has the highest optical depth, and is therefore, an excellent tracer of outflow and infall motions that give rise to non-Gaussian profiles.

For each clump, we determine a single $V_{\mathrm{C}}$ to describe its associated line emission. Deriving a $V_{\mathrm{C}}$ is important since it will be used to determine kinematic distances to each clump (Whitaker et al. in preparation) and, hence, their masses and luminosities (Contraras et al. in preparation). The difference between individually derived velocities and the $V_{\mathrm{C}}$ within 


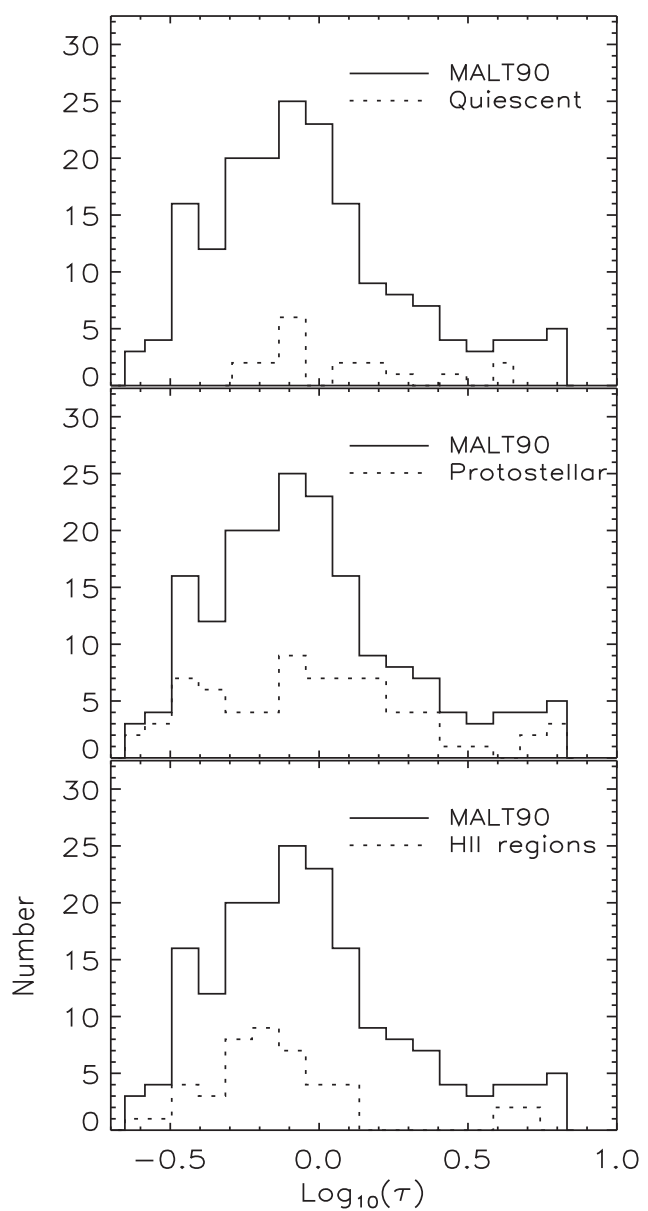

Figure 21. Histograms of the measured opacities for clumps located outside of the CMZ (i.e., $\pm 10^{\circ}$ of the Galactic centre). In all panels, the solid histogram shows the distribution derived from the full survey, while the dotted histograms show the distributions for each of the IR-based categories. We find that the distribution of $\tau$ from the full survey has a median value of $\sim 0.8$. The differences in the overall distributions for the quiescent clumps and $\mathrm{H}_{\mathrm{II}}$ regions indicates a trend of decreasing opacity with increasing evolutionary stage.

individual clumps reveals that despite the exact excitation conditions, most of the line emission is generally well centred around the $V_{\mathrm{C}}$. This implies that the emission from the various species arise from within the same physical regions.

We broadly classify the clumps into groups based on their IR signatures (see Jackson et al. 2013; Guzmán et al. 2015). We find that clumps with active star formation (i.e., those classified as either protostellar or $\mathrm{H}_{\text {II }}$ regions), have a higher percentage of detections for most emission lines compared to the full survey and those clumps that appear to be more quiescent. Moreover, the protostellar clumps and $\mathrm{H}_{\text {II }}$ regions are detected in four or more lines more frequently ( $\sim 62$ and $\sim 58 \%$, respectively) compared to the full survey $(\sim 53 \%)$ and the quiescent clumps ( $\sim 38 \%)$.

We find several interesting trends in the ensemble of properties for the different molecular transitions when plotted as a function of the clumps' evolutionary state. From trends in the II ratios, we find that (1) HNC is relatively brighter in colder, less evolved clumps than those that show active star-formation, (2) $\mathrm{N}_{2} \mathrm{H}^{+}$is relatively brighter in the earlier stages, and (3) that the observed optical depth decreases as the clumps evolve. Trends in the velocity differences show a particularly striking result: The optically thickest $\mathrm{HCO}^{+}$emission shows a 'blue-red asymmetry' that indicates overall collapse that monotonically decreases as the clumps evolve.

This catalogue represents the largest compiled database of line emission towards high-mass clumps. As such, it is a valuable data set for studies of these clumps and provides a definitive source list for ALMA observations of the smallscale structure within high-mass clumps.

\section{ACKNOWLEDGEMENTS}

The MALT90 project team gratefully acknowledges the use of dense clump positions supplied by ATLASGAL. ATLASGAL is a collaboration between the Max Planck Gesellschaft (MPG: Max Planck Institute for Radioastronomy, Bonn and the Max Planck Institute for Astronomy, Heidelberg), the European Southern Observatory (ESO) and the University of Chile. This work was supported by NSF grant AST-1211844. A.E.G. acknowledges support of CONICYT through FONDECYT 3150570.

Facilities: Mopra.

\section{REFERENCES}

Aguirre, J. E., et al. 2011, ApJS, 192, 4

Ao, Y., et al. 2013, A\&A, 550, A135

Benjamin, R. A., et al. 2003, PASP, 115, 953

Carey, S. J., et al. 2009, PASP, 121, 76

Caswell, J. L., et al. 2010, MNRAS, 404, 1029

Churchwell, E., et al. 2009, PASP, 121, 213

Contreras, Y., Garay, G., Rathborne, J. M., \& Sanhueza, P. 2016, MNRAS, 456, 2041

Contreras, Y., et al. 2013, A\&A, 549, A45

Csengeri, T., et al. 2016, A\&A, 586, A149

Foster, J. B., et al. 2011, ApJS, 197, 25

Foster, J. B., et al. 2013, PASA, 30, 38

Green, J. A., et al. 2009, MNRAS, 392, 783

Guzmán, A. E., Sanhueza, P., Contreras, Y., Smith, H. A., Jackson, J. M., Hoq, S., \& Rathborne, J. M. 2015, ApJ, 815, 130

He, Y.-X., et al. 2015, MNRAS, 450, 1926

Hoare, M. G., et al.2005, in IAU Symp., Vol. 227, Massive Star Birth: A Crossroads of Astrophysics, eds. R. Cesaroni, M. Felli, E. Churchwell, \& M. Walmsley (Cambridge: Cambridge University Press), 370

Hoare, M. G., et al. 2012, PASP, 124, 939

Hoq, S., et al. 2013, ApJ, 777, 157

Jackson, J. M., et al. 2013, PASA, 30, 57

Ladd, N., Purcell, C., Wong, T., \& Robertson, S. 2005, PASA, 22, 62

Liu, X.-L., Wang, J.-J., \& Xu, J.-L. 2013, MNRAS, 431, 27

Lumsden, S. L., Hoare, M. G., Urquhart, J. S., Oudmaijer, R. D., Davies, B., Mottram, J. C., Cooper, H. D. B., \& Moore, T. J. T. 2013, ApJS, 208, 11

Martín-Pintado, J., Rizzo, J. R., de Vicente, P., RodríguezFernández, N. J., \& Fuente, A. 2001, ApJ, 548, L65 
Miettinen, O. 2014, A\&A, 562, A3

Molinari, S., et al. 2010, PASP, 122, 314

Müller, H. S. P., Schlöder, F., Stutzki, J., \& Winnewisser, G. 2005, JMoSt, 742, 215

Müller, H. S. P., Thorwirth, S., Roth, D. A., \& Winnewisser, G. 2001, A\&A, 370, L49

Purcell, C. R., et al. 2013, ApJS, 205, 1

Purcell, C. R., et al. 2012, MNRAS, 426, 1972

Rathborne, J. M., et al. 2015, ApJ, 802, 125

Rathborne, J. M., et al. 2014a, ApJ, 786, 140

Rathborne, J. M., et al. 2014b, ApJ, 795, L25

Sanhueza, P., Jackson, J. M., Foster, J. B., Garay, G., Silva, A., \& Finn, S. C. 2012, ApJ, 756, 60

Schöier, F. L., van der Tak, F. F. S., van Dishoeck, E. F., \& Black, J. H. 2005, A\&A, 432, 369

Schuller, F., et al. 2009, A\&A, 504, 415

Shirley, Y. L., Nordhaus, M. K., Grcevich, J. M., Evans, II, N. J., Rawlings, J. M. C., \& Tatematsu, K. 2005, ApJ, 632, 982
Simpson, G., \& Mayer-Hasselwander, H. 1986, A\&A, 162, 340

Stephens, I. W., Jackson, J. M., Sanhueza, P., Whitaker, J. S., Hoq, S., Rathborne, J. M., \& Foster, J. B. 2015, ApJ, 802, 6

Tremblay, C. D., Walsh, A. J., Longmore, S. N., Urquhart, J. S., \& König, C. 2015, PASA, 32, e047

Urquhart, J. S., et al. 2014a, A\&A, 568, A41

Urquhart, J. S., Hoare, M. G., Lumsden, S. L., Oudmaijer, R. D., \& Moore, T. J. T. 2008, in ASP Conf. Ser., Vol. 387, Massive Star Formation: Observations Confront Theory, eds. H. Beuther, H. Linz, \& T. Henning (San Francisco: Astron. Soc. Pac.), 381

Urquhart, J. S., et al. 2014b, MNRAS, 443, 1555

Urquhart, J. S., et al. 2013, MNRAS, 431, 1752

Walmsley, C. M., Guesten, R., Angerhofer, P., Churchwell, E., \& Mundy, L. 1986, A\&A, 155, 129

Walsh, A. J., et al. 2011, MNRAS, 416, 1764

Wilson, T. L., Ruf, K., Walmsley, C. M., Martin, R. N., Batrla, W., \& Pauls, T. A. 1982, A\&A, 115, 185

Yu, N., \& Wang, J.-J. 2015, MNRAS, 451, 2507 